\title{
AN ANALYSIS TECHNIQUE FOR STABILIZED FINITE ELEMENT SOLUTION OF INCOMPRESSIBLE FLOWS *
}

\author{
Tomás Chacón ReBollo ${ }^{1}$
}

\begin{abstract}
This paper presents an extension to stabilized methods of the standard technique for the numerical analysis of mixed methods. We prove that the stability of stabilized methods follows from an underlying discrete inf-sup condition, plus a uniform separation property between bubble and velocity finite element spaces. We apply the technique introduced to prove the stability of stabilized spectral element methods so as stabilized solution of the primitive equations of the ocean.
\end{abstract}

Mathematics Subject Classification. 65N30, 76M10.

Received: November 24, 1999. Revised: October 19, 2000.

\section{INTRODUCTION AND MOTIVATION}

This paper deals with the numerical analysis of the solution of incompressible flow problems by stabilized finite elements. We shall be interested in the Oseen equations (Stokes equations plus a linear transport term), also called in some works "linearized Navier-Stokes equations".

Stabilized methods provide efficient and computationally cheap techniques to solve incompressible fluids. Historically, these methods have been the object of a specific analysis, different from that of mixed methods. Indeed, the proof of stability is not based upon the existence of a discrete velocity - pressure inf-sup condition, but rather upon specific arguments that strongly rely on the elementwise regularity of finite element functions. Based upon such kind of arguments, the papers of Hugues, Franca and Balestra [21] and Hughes and Franca [20] contained an error analysis that was improved in Brezzi and Douglas [8] and in Pierre [25]. In Franca and Stenberg [15] a general stability and error analysis technique was introduced, which was summarized in Franca, Hugues and Stenberg [16]. Also, the paper of Tobiska and Verfürth [27] develops an analysis of stability and convergence for the solution of Navier-Stokes equations by stabilized methods.

Another way of analysis is suggested by the relationship between stabilized and mixed methods. In Franca and Frey [14] it is proved that the Streamline Upwind/Petrov-Galerkin (SUPG) method is equivalent to the standard mixed method constructed with the mini-element. This equivalence is understood in the sense that both methods yield the same formulation if the degrees of freedom associated to the bubbles are eliminated by static condensation. This equivalence yields the stability of SUPG method from that of the mixed method

\footnotetext{
Keywords and phrases. Oseen equations, finite elements, mixed methods, stabilized methods, discrete inf-sup condition, spectral methods, primitive equations.

* This research was partially supported by Spanish M. E. C. Project MAR97-1055-C02-02 and by EU HCM ERBCHB ICT 94.1823 Grant.

${ }^{1}$ Departamento de Ecuaciones Diferenciales y Análisis Numérico, Universidad de Sevilla. C/ Tarfia, s/n. 41080 Sevilla, Spain. e-mail: chacon@numer.us.es
} 
constructed with the mini-element. It is a direct consequence of the fact that this element satisfies the discrete inf-sup condition. Such analysis is essentially performed in Chacón Rebollo [10].

We address in this paper the question of whether this way of analysis may be applied to stabilized methods other than SUPG. We develop a technique for the numerical analysis of stabilized methods that gives a positive answer to that question. Concretely, we prove the existence of an underlying discrete inf-sup condition from which we deduce the stability of stabilized methods. Once this point has been set up, our technique allows to analyze stabilized methods as if they where mixed methods (Th. 1). They appear as internal approximations of a weak formulation, whose stability relies on an inf-sup condition. Then, our analysis may be applied to more complex situations, where we use the tools provided by functional analysis to obtain gains with respect to the standard analysis. We include in this paper two of such applications:

- To prove the stability of a spectral element approximation of the generalized Stokes equations, introduced in Gervasio and Saleri [19]. Here, we obtain $L^{2}$ estimates for the pressure, while the standard analysis, used in that paper, allows only to estimate a seminorm of the pressure gradient.

- To solve a linear model of primitive equations of the ocean by stabilized finite elements. For such equations, there is some lack of regularity for the convection term, so that the pressure has only $L^{p}$ regularity, for some $p \in(1,2)$. In this case, we obtain $L^{p}$ estimates for the discrete pressure, and prove convergence in $H^{1} \times L^{p}$ norm to the continuous solution. The standard analysis in this context would be quite difficult to be carried on.

Our analysis may also be applied to nonlinear flows. For instance, in Chacón Rebollo and Domínguez Delgado [11], it is applied to the analysis of the approximation of Navier-Stokes equations by stabilized methods, in parallel to the analysis of their approximations by mixed methods. Stability and error estimates are derived. This analysis also applies to nonlinear stabilized methods, such as the optimal one introduced in Russo [26]. Up to our knowledge, the standard analysis is unable to handle nonlinear stabilization, which turns out to be rather simple to manage with our technique.

Another possible application is the analysis of the solution of Oseen equations by the reduced $Q_{1} / Q_{1}$ stabilized methods introduced in Knobloch and Tobiska [22]. This is a new family of computationally cheap methods that may be directly analyzed with our analysis. In fact, all hypothesis of Theorem 1 are readily proved to be satisfied, using the analysis developped in that paper.

We would like to point out that the analysis technique that we introduce is rather complex from a technical point of view. However, we think that it is worth to be used, as it essentially reduces the difficulties of the analysis of stabilized methods to that of mixed method. Moreover, we have tried to present the technique in a systematic way, so that it may be applied to situations other than the considered here, with relative ease.

The paper is organized as follows. In Section 2 we introduce an abstract discretization of Oseen equations, whose stability is analyzed in Section 3. In Section 4, we apply the abstract theory to stabilized methods. Section 5 is devoted to the analysis of spectral element stabilized methods. Finally, in Section 6 we solve a linear model of primitive equations of the ocean by stabilized finite elements.

\section{Abstract Discretization}

In this Section we introduce an abstract discretization of Oseen equations which is the base of our analysis.

Let us consider a connected bounded domain $\Omega \subset \mathbf{R}^{d}(d=2$ or 3$)$, with Lipschitz-continuous boundary $\Gamma$. We are given a "driving" velocity field $\mathbf{u}: \Omega \longrightarrow \mathbf{R}^{d}$, that we assume to be divergence-free. Our purpose is to solve numerically the following boundary value problem:

$$
\left\{\begin{array}{cl}
\text { Find } \mathbf{y}: \Omega \longrightarrow \mathbf{R}^{d}, \quad p: \Omega \longrightarrow \mathbf{R} & \text { such that } \\
\mathbf{u} \cdot \nabla \mathbf{y}-\nu \Delta \mathbf{y}+\nabla p=\mathbf{f}, \quad \nabla \cdot \mathbf{y}=0 & \text { in } \Omega, \\
\mathbf{y}=0 & \text { on } \Gamma
\end{array}\right.
$$


Here, $\nu>0$ is the viscosity coefficient, and $\mathbf{f} \in\left[H^{-1}(\Omega)\right]^{d}$ is a given source term. Only homogeneous Dirichlet boundary conditions are considered, in order to not introduce nonessential difficulties in our derivation.

Let us define the bilinear form on $\left[H_{0}^{1}(\Omega)\right]^{d} \times\left[H_{0}^{1}(\Omega)\right]^{d}$,

$$
a(\mathbf{w}, \mathbf{v})=(\mathbf{u} \cdot \nabla \mathbf{w}, \mathbf{v})+\nu(\nabla \mathbf{w}, \nabla \mathbf{v}), \quad \forall \mathbf{w}, \mathbf{v} \in\left[H_{0}^{1}(\Omega)\right]^{d}
$$

where we denote by $(\cdot, \cdot)$ the $L^{2}$ scalar product, either for scalar, vector or tensor functions. If we assume that $\mathbf{u} \in\left[L^{p}(\Omega)\right]^{d}$ for some $p>d$, and $\nabla \cdot \mathbf{u}=0$, then $a(\cdot, \cdot)$ is well defined and is continuous and $\left[H_{0}^{1}(\Omega)\right]^{d}$-elliptic; i.e., it verifies

$$
a(\mathbf{w}, \mathbf{v}) \leq \mathcal{M}(\mathbf{u})|\mathbf{w}|_{1}|\mathbf{v}|_{1}, a(\mathbf{v}, \mathbf{v}) \geq \nu|\mathbf{v}|_{1}^{2} \quad \forall \mathbf{v}, \mathbf{w} \in\left[H_{0}^{1}(\Omega)\right]^{d}
$$

Here, we have denoted by $|\cdot|_{1}$ the $\left[H^{1}(\Omega)\right]^{d}$ seminorm. Also, $\mathcal{M}(\mathbf{u})=C\left(\|\mathbf{u}\|_{0, p}+\nu\right)$ for some constant $C$ appearing from Sobolev injections, where $\|\cdot\|_{0, p}$ denotes the $L^{p}$ norm.

The form $a(\cdot, \cdot)$ defines a linear bounded operator $\mathcal{A}$ from $\left[H_{0}^{1}(\Omega)\right]^{d}$ into $\left[H^{-1}(\Omega)\right]^{d}$, given by

$$
\langle\mathcal{A} \mathbf{w}, \mathbf{v}\rangle=a(\mathbf{w}, \mathbf{v}), \quad \forall \mathbf{w}, \mathbf{v} \in\left[H_{0}^{1}(\Omega)\right]^{d}
$$

Thus, $\mathcal{A} \mathbf{w}=\mathbf{u} \cdot \nabla \mathbf{w}-\nu \Delta \mathbf{w}$.

The standard mixed formulation of problem (1) reads as follows:

$$
\left\{\begin{array}{l}
\text { Obtain }(\mathbf{y}, p) \in\left[H_{0}^{1}(\Omega)\right]^{d} \times L_{0}^{2}(\Omega) \text { such that } \\
B(\mathbf{y}, p ; \mathbf{v}, q)=\langle\mathbf{f}, \mathbf{v}\rangle, \quad \forall(\mathbf{v}, q) \in\left[H_{0}^{1}(\Omega)\right]^{d} \times L_{0}^{2}(\Omega)
\end{array}\right.
$$

where

$$
B(\mathbf{y}, p ; \mathbf{v}, q)=a(\mathbf{y}, \mathbf{v})-(p, \nabla \cdot \mathbf{v})-(\nabla \cdot \mathbf{y}, q) .
$$

Also, $\langle\cdot, \cdot\rangle$ stands for the $\left[H^{-1}(\Omega)\right]^{d}-\left[H_{0}^{1}(\Omega)\right]^{d}$ duality, and $L_{0}^{2}(\Omega)$ is the subspace of $L^{2}(\Omega)$ given by

$$
L_{0}^{2}(\Omega)=\left\{q \in L^{2}(\Omega) \text { such that } \int_{\Omega} q \mathrm{~d} x=0\right\} .
$$

The pair of spaces $\left(\left[H_{0}^{1}(\Omega)\right]^{d}, L_{0}^{2}(\Omega)\right)$ verifies the continuous inf-sup condition (cf. Girault and Raviart [17]). Then, due to properties (3), problem (4) has a unique solution that depends continuously on the data $\mathbf{f}$.

In order to describe our abstract discretization of problem (4) we shall consider two families of subspaces $\left\{Y_{h}\right\}_{h>0}$ and $\left\{Z_{h}\right\}_{h>0}$ of $\left[H_{0}^{1}(\Omega)\right]^{d}$ and another family of subspaces $\left\{M_{h}\right\}_{h>0}$ of $L_{0}^{2}(\Omega)$, all of them of finite dimension. These spaces may be, for instance, standard finite element spaces. We shall also consider a family of bilinear continuous forms on $\left[H_{0}^{1}(\Omega)\right]^{d} \times\left[H_{0}^{1}(\Omega)\right]^{d},\left\{\mathbf{S}_{h}(\cdot, \cdot)\right\}_{h>0}$. These forms are assumed to be coercive in $H^{1}$ norm on $Z_{h}$.

We shall denote by $\mathcal{R}_{h}$ the "static condensation" operator

$$
\mathcal{R}_{h}:\left[H^{-1}(\Omega)\right]^{d} \rightarrow Z_{h}
$$

defined as follows. Given $\varphi \in\left[H^{-1}(\Omega)\right]^{d}, \mathcal{R}_{h}(\varphi)$ is the only element of $Z_{h}$ that satisfies

$$
\mathbf{S}_{h}\left(\mathcal{R}_{h}(\varphi), \mathbf{z}_{h}\right)=\left\langle\varphi, \mathbf{z}_{h}\right\rangle, \quad \forall \mathbf{z}_{h} \in Z_{h}
$$


We discretize problem (4) by

$$
\left\{\begin{array}{l}
\text { Obtain }\left(\mathbf{y}_{h}, p_{h}\right) \in Y_{h} \times M_{h} \text { such that } \\
B_{h}\left(\mathbf{y}_{h}, p_{h} ; \mathbf{v}_{h}, q_{h}\right)=F_{h}\left(\mathbf{v}_{h}, q_{h}\right), \quad \forall\left(\mathbf{v}_{h}, q_{h}\right) \in Y_{h} \times M_{h} ;
\end{array}\right.
$$

where

$$
\begin{aligned}
B_{h}(\mathbf{w}, r ; \mathbf{v}, q) & =B(\mathbf{w}, r ; \mathbf{v}, q)-\mathbf{S}_{h}\left(\mathcal{R}_{h}(\mathcal{B} \mathbf{v}+\nabla q), \mathcal{R}_{h}(\mathcal{A} \mathbf{w}+\nabla r)\right) \\
F_{h}(\mathbf{v}, q) & =\langle\mathbf{f}, \mathbf{v}\rangle-\mathbf{S}_{h}\left(\mathcal{R}_{h}(\mathcal{B} \mathbf{v}+\nabla q), \mathcal{R}_{h}(\mathbf{f})\right)
\end{aligned}
$$

where $\mathcal{B}$ denotes the operator

$$
\mathcal{B} \mathbf{w}=-\mathbf{u} \cdot \nabla \mathbf{w}+\varepsilon \nu \Delta \mathbf{w}, \quad \forall \mathbf{w} \in\left[H_{0}^{1}(\Omega)\right]^{d}
$$

for a given $\varepsilon \in \mathbf{R}$.

We shall use method (6) as an abstract framework to analyze various standard stabilized methods. To describe these methods, we shall consider affine-equivalent finite element spaces, as described in Hughes, Franca and Balestra [21]. Assume that the domain $\Omega$ is polyhedric. Let us consider a triangulation $\mathcal{T}_{h}$ of $\Omega$ formed by either simplicial or parallelepipedic elements. We assume that the elements of $\mathcal{T}_{h}$ are affine-transformed of a reference element $K^{*}$ (either the unit simplex or parallelepiped), in the sense of Ciarlet [13]. Given an integer number $k \geq 0$, and an element $K \in \mathcal{T}_{h}$, denote by $P_{k}(K)$ the space of polynomials of degree smaller than, or equal to, $k$, defined on $K$. Also, denote by $Q_{k}(K)$ the space of polynomials of degree smaller than, or equal to, $k$, in each variable, defined on $K$. Denote by $R_{k}(K)$ either $P_{k}(K)$, if $K$ is a triangle or tetrahedron, or $Q_{k}(K)$ if $K$ is a quadrilateral or hexaedron. Given two integer numbers $m \geq 1, l \geq 0$, consider the following finite element spaces.

$$
\begin{gathered}
Y_{h}^{(m)}=\left\{\mathbf{v} \in\left[H_{0}^{1}(\Omega)\right]^{d} \mid \mathbf{v}_{\left.\right|_{K}} \in\left[R_{m}(K)\right]^{d}, \forall K \in \mathcal{T}_{h}\right\} ; \\
M_{h}^{(l)}=\left\{q \in L_{0}^{2}(\Omega) \mid q_{\left.\right|_{K}} \in R_{l}(K), \forall K \in \mathcal{T}_{h}\right\},
\end{gathered}
$$

or

$$
M_{h}^{(l)}=\left\{q \in L_{0}^{2}(\Omega) \cap C^{0}(\Omega) \mid q_{\left.\right|_{K}} \in R_{l}(K), \forall K \in \mathcal{T}_{h}\right\}
$$

We consider the following stabilized methods.

$$
\left\{\begin{array}{l}
\text { Find }\left(\mathbf{y}_{h}, p_{h}\right) \in Y_{h}^{(m)} \times M_{h}^{(l)} \text { such that } \\
B_{S}\left(\mathbf{y}_{h}, p_{h} ; \mathbf{v}_{h}, q_{h}\right)=F_{S}\left(\mathbf{v}_{h}, q_{h}\right), \quad \forall\left(\mathbf{v}_{h}, q_{h}\right) \in Y_{h}^{(m)} \times M_{h}^{(l)} ;
\end{array}\right.
$$

where

$$
\begin{aligned}
B_{S}(\mathbf{w}, r ; \mathbf{v}, q) & =B(\mathbf{w}, r ; \mathbf{v}, q)-\sum_{K \in \mathcal{T}_{h}} \tau_{K}(\mathcal{B} \mathbf{v}+\nabla q ; \mathcal{A} \mathbf{w}+\nabla r)_{K} \\
F_{S}(\mathbf{v}, q) & =\langle\mathbf{f}, \mathbf{v}\rangle-\sum_{K \in \mathcal{T}_{h}} \tau_{K}(\mathcal{B} \mathbf{v}+\nabla q, \mathbf{f})_{K}
\end{aligned}
$$

where the $\tau_{K}$ are given stabilizing coefficients, and $(\cdot, \cdot)_{K}$ denotes the inner product in $\left[L^{2}(K)\right]^{d}$. When $l=m=1$, method (10) is independent of the actual value of the coefficient $\varepsilon$, and it is known as Streamline 
Upwind/Petrov-Galerkin (SUPG) method. For other values of $m \geq 1$ and $l \geq 0$, when $\varepsilon=-1,0$ and 1 , method (10) is respectively known as Adjoint stabilized (AdS), generalized SUPG and Galerkin-Least Squares (GaLS) method.

Typically, the coefficients $\tau_{K}$ are continuous functions of the local Péclet number on element $K$,

$$
\begin{gathered}
P e_{K}=\frac{\mathbf{U}_{K} h_{K}}{\nu} \quad \text { with } \quad \mathbf{U}_{K}=\left[\int_{K}|\mathbf{u}|^{p}\right]^{1 / p} ; \\
\tau_{K}\left(P e_{K}\right)=A \frac{h_{K}}{\mathbf{U}_{K}} \min \left(P e_{K}, P\right)= \begin{cases}A \frac{h_{K}^{2}}{\nu} & \text { if } P e_{K} \leq P, \\
A P \frac{h_{K}}{\mathbf{U}_{K}} & \text { if } P e_{K}>P ;\end{cases}
\end{gathered}
$$

where $A$ is a numerical constant and $P$ is a preset threshold for the Péclet number. This allows on one hand to introduce some suitable stabilization of high frequence components of the transport operator (of order $h_{K}$ ), due to convection dominance (Large $P e_{K}$ ). Also, this introduces low levels of numerical diffusion (of order $h_{K}^{2}$ ) in regions where diffusion is dominant (Low $P e_{K}$ ). On the other hand, this stabilizes the spurious modes of the pressure gradient.

Also, for reasons of computability, in practice the convection velocity $\mathbf{u}$ is replaced in the stabilizing terms by some stable interpolate $\mathbf{u}_{h} \in Y_{h}^{(m)}$. We shall assume it so in our analysis.

The standard analysis of stabilized methods, summarized in Franca, Hughes and Stenberg [16], states that SUPG and GaLS methods are stable for any positive coefficients $\tau_{K}$, and that AdS and generalized SUPG methods are stable if the $\tau_{K}$ are small enough. The obtention of optimal bounds for these coefficients to ensure stability requires the computation of the best constant $C_{I}$ in the inverse inequality

$$
C_{I} \sum_{K \in \mathcal{T}_{h}} h_{K}^{2}\left\|\Delta \mathbf{v}_{h}\right\|_{K}^{2} \leq\left\|\nabla \mathbf{v}_{h}\right\|_{0}^{2}, \quad \forall \mathbf{v}_{h} \in Y_{h}^{(m)}
$$

That analysis applies to either continuous pressures combined with velocities of arbitrary interpolation degree, or to discontinuous pressures combined with high-degree interpolation velocities. Concretely, it holds under the following condition:

$$
\text { Either } M_{h}^{(l)} \subset C^{0}(\bar{\Omega}) \text {, or } m \geq n
$$

where

$$
n= \begin{cases}d & \text { if } \mathcal{T}_{h} \text { is formed by triangles or tetrahedra, and } \\ 2 & \text { if } \mathcal{T}_{h} \text { is formed by quadrilaterals or hexaedra. }\end{cases}
$$

In Tobiska and Verfürth [27] this restriction is removed by introducing in the structure of the method some additional terms that take into account interelement pressure jump terms. However, it seems that method (10), without these jump terms, is not able to stabilize the discretization of discontinuous pressures combined with low-degree velocities.

In this paper we shall analyze methods satisfying condition (13). Our analysis also applies to general discretizations that do not necessarily satisfy this condition. However, its proof requires a rather lengthy derivation that shall appear in a forthcoming paper.

Notice that method (6) applies to general internal approximations of $\left[H_{0}^{1}(\Omega)\right]^{d}$ and $L_{0}^{2}(\Omega)$, while stabilized methods only apply to approximations by piecewise smooth functions. We are, thus, considering a genuine generalization of stabilized methods.

In the next two Sections we first develop a stability and convergence analysis for the abstract method (6) which extends the standard analysis of mixed methods, and next apply it to analyze the stabilized methods (10). 


\section{Analysis of abstract method}

In this section we prove that the stability of the abstract method (6) follows from a discrete inf-sup BrezziBabuška condition, similarly to mixed methods.

The stability of abstract method (6), in addition to the inf-sup condition, requires the following hypotheses on the new elements appearing in method (6):

Hypothesis 1. There exists a constant $C_{0}>0$ independent of $h$ such that

$$
\left|\mathbf{y}_{h}\right|_{1}+\left|\mathbf{z}_{h}\right|_{1} \leq C_{0}\left|\mathbf{y}_{h}+\mathbf{z}_{h}\right|_{1}, \quad \forall \mathbf{y}_{h} \in Y_{h}, \mathbf{z}_{h} \in Z_{h}, \quad \forall h>0
$$

Hypothesis 2. There exist two constants $\nu_{s}>0, M_{s}>0$ such that

$$
\left|\mathbf{S}_{h}\left(\mathbf{w}_{h}, \mathbf{v}_{h}\right)\right| \leq M_{s}\left|\mathbf{w}_{h}\right|_{1}\left|\mathbf{v}_{h}\right|_{1}, \mathbf{S}_{h}\left(\mathbf{v}_{h}, \mathbf{v}_{h}\right) \geq \nu_{s}\left|\mathbf{v}_{h}\right|_{1}^{2}, \quad \forall \mathbf{w}_{h}, \mathbf{v}_{h} \in Z_{h}
$$

Both hypotheses play a crucial role in the obtention of estimates for both velocity and pressure, and thus in the proof of stability of method (6). Hypothesis 1 is a generalization of the well known $H_{0}^{1}$-orthogonality between piecewise affine and bubble finite elements. Hypothesis 2 is a generalization of the fact that the stabilizing coefficients in (11) are of order $h_{K}^{2}$.

Let us recall the definition of stability for method (6) (cf. Babuška [3], Brezzi [7]):

Definition 1. Method (6) is said to be stable on $Y_{h} \times M_{h}$ if there is a constant $\gamma>0$ independent of $h$ such that for any $(\mathbf{w}, r) \in Y_{h} \times M_{h}$,

$$
\begin{array}{lll} 
& \sup _{(\mathbf{v}, q)} \in Y_{h} \times M_{h} & \frac{B_{h}(\mathbf{w}, r ; \mathbf{v}, q)}{|\mathbf{v}|_{1}+\|q\|_{0}} \geq \gamma\left(|\mathbf{w}|_{1}+\|r\|_{0}\right) \\
(\mathbf{v}, q) & \neq & \\
& & \\
& \sup _{(0,0)} & \frac{B_{h}(\mathbf{v}, q ; \mathbf{w}, r)}{|\mathbf{v}|_{1}+\|q\|_{0}} \geq \gamma\left(|\mathbf{w}|_{1}+\|r\|_{0}\right) \\
(\mathbf{v}, q) & \in Y_{h} \times M_{h} & \\
(\mathbf{v}, q) & \neq & (0,0)
\end{array}
$$

We now state our basic stability result.

Theorem 1. Assume that the pairs of spaces $\left\{\left(Y_{h}+Z_{h}, M_{h}\right)\right\}_{h>0}$ satisfy a uniform discrete Brezzi-Babuška condition, and that Hypotheses 1 and 2 hold. Assume that at least one of the two following sentences hold:

i) $Z_{h}$ and $Y_{h}$ are orthogonal with respect to the $\left[H_{0}^{1}(\Omega)\right]^{d}$ inner product and $\nu_{s}>0$, or

ii) $\nu_{s} \geq\left(\frac{1-\varepsilon}{2}\right)^{2} \nu$, when $\varepsilon \neq 1$, or $\nu_{s}>0$ when $\varepsilon=1$.

Then, the abstract method (6) is stable.

From this theorem we deduce the main result of this paper:

Theorem 2. Assume that the family of triangulations $\left\{\mathcal{T}_{h}\right\}_{h>0}$ is regular. Assume that condition (13) holds. Then, the stabilized method (10) coincides with an abstract method (6) constructed with a finite element space $Z_{h}$ of bubble functions and a bilinear form $S_{h}$, verifying

1. The pairs of spaces $\left\{Y_{h}+Z_{h}, M_{h}\right\}_{h>0}$ satisfy a uniform discrete inf-sup condition.

2. The pairs of spaces $\left\{Y_{h}, Z_{h}\right\}_{h>0}$ satisfy Hypothesis 1.

3. The forms $\left\{S_{h}\right\}_{h>0}$ satisfy Hypothesis 2. 
As a consequence,

- GaLS and SUPG methods are stable for any $A>0$ in (11)

- The general stabilized method (10) is stable if $A \leq A_{0}\left(\frac{2}{\varepsilon-1}\right)^{2}$, where $A_{0}$ is a computable positive constant. In particular, AdS method is stable if $A \leq A_{0}$, and generalized SUPG method is stable if $A \leq 4 A_{0}$.

Thus, under our analysis, the stability of stabilized methods follows from a discrete inf-sup condition, similarly to mixed methods. We shall prove this result in Section 4. In addition, we shall prove that the constant $A_{0}$ depends on the aspect ratio of the grid and on the reference elements of spaces $Y_{h}$ and $M_{h}$, and shall give computable fine estimates for this constant.

Proof of Theorem 1.

Velocity estimate. We shall treat separately cases i) and ii).

i) Assume that spaces $Z_{h}$ and $Y_{h}$ are orthogonal with respect to the $\left[H_{0}^{1}(\Omega)\right]^{d}$ inner product. In this case, all methods (10) coincide, independently of the actual value of $\varepsilon$, as such orthogonality implies $\mathcal{R}_{h}(\Delta \mathbf{w})=0, \forall \mathbf{w} \in$ $Y_{h}$.

Consider a pair $\left(\mathbf{w}_{h}, r_{h}\right) \in Y_{h} \times M_{h}$. Define $\mathbf{c}_{h}=\mathcal{R}_{h}\left(\mathcal{A} \mathbf{w}_{h}+\nabla r_{h}\right)$. As $\mathcal{R}_{h}\left(\Delta \mathbf{v}_{h}\right)=0$, then $\mathbf{c}_{h}=\mathcal{R}_{h}\left(-\mathcal{B} \mathbf{w}_{h}+\nabla r_{h}\right)$. Consequently,

$$
B_{h}\left(\mathbf{w}_{h}, r_{h} ; \mathbf{w}_{h},-r_{h}\right)=a\left(\mathbf{w}_{h}, \mathbf{w}_{h}\right)+\mathbf{S}_{h}\left(\mathbf{c}_{h}, \mathbf{c}_{h}\right) \geq \nu\left|\mathbf{w}_{h}\right|_{1}^{2}+\nu_{s}\left|\mathbf{c}_{h}\right|_{1}^{2} .
$$

ii) Consider a pair $\left(\mathbf{w}_{h}, r_{h}\right) \in Y_{h} \times M_{h}$. Define $\mathbf{c}_{h}=\mathcal{R}_{h}\left(\mathcal{A} \mathbf{w}_{h}+\nabla r_{h}\right)$. Then,

$$
\begin{aligned}
B\left(\mathbf{w}_{h}, r_{h} ; \mathbf{w}_{h},-r_{h}\right) & =a\left(\mathbf{w}_{h}, \mathbf{w}_{h}\right)+\mathbf{S}_{h}\left(\mathbf{c}_{h}, \mathbf{c}_{h}\right)+(1-\varepsilon) \nu \mathbf{S}_{h}\left(\mathcal{R}_{h}\left(\Delta \mathbf{w}_{h}\right), \mathbf{c}_{h}\right) \\
& =a\left(\mathbf{w}_{h}, \mathbf{w}_{h}\right)+\mathbf{S}_{h}\left(\mathbf{c}_{h}, \mathbf{c}_{h}\right)-(1-\varepsilon) \nu\left(\nabla \mathbf{w}_{h}, \nabla \mathbf{c}_{h}\right)
\end{aligned}
$$

Due to Hypothesis 1,

$$
\left|\left(\nabla \mathbf{y}_{h}, \nabla \mathbf{z}_{h}\right)\right| \leq\left(1-\delta_{0}\right)\left|\mathbf{y}_{h}\right|_{1}\left|\mathbf{z}_{h}\right|_{1}, \quad \forall \mathbf{y}_{h} \in Y_{h}, \mathbf{z}_{h} \in Z_{h}, \text { where } \delta_{0}=\frac{2}{C_{0}^{2}} .
$$

Then, using Young's inequality, (15) implies

$$
B\left(\mathbf{w}_{h}, r_{h} ; \mathbf{w}_{h},-r_{h}\right) \geq \tilde{\nu}\left|\mathbf{w}_{h}\right|_{1}^{2}+\tilde{\nu}_{s}\left|\mathbf{c}_{h}\right|_{1}^{2},
$$

where

$$
\tilde{\nu}=\nu\left[1-\left(1-\delta_{0}\right) \frac{|1-\varepsilon|}{2} \mu\right], \quad \tilde{\nu}_{s}=\nu_{s}-\nu\left(1-\delta_{0}\right) \frac{|1-\varepsilon|}{2} \mu^{-1}
$$

for any $\mu>0$. When $\varepsilon=1, \tilde{\nu}=\nu$ and $\tilde{\nu}_{s}=\nu_{s}>0$. When $\varepsilon \neq 1$, we may choose

$$
\frac{\nu}{\nu_{s}} \frac{|1-\varepsilon|}{2}\left(1-\delta_{0}\right)<\mu<\frac{2}{|1-\varepsilon|}\left(1-\delta_{0}\right)^{-1}
$$

and then $\tilde{\nu}>0, \tilde{\nu}_{s}>0$.

Denote

Then, in all cases

$$
S=\underset{(\mathbf{v}, q) \quad \sup _{(\mathbf{v}, q)} \neq Y_{h} \times M_{h}}{ } \frac{B_{h}\left(\mathbf{w}_{h}, r_{h} ; \mathbf{v}, q\right)}{|\mathbf{v}|_{1}+\|q\|_{0}} .
$$

$$
\tilde{\nu}\left|\mathbf{w}_{h}\right|_{1}^{2}+\tilde{\nu}_{s}\left|\mathbf{c}_{h}\right|_{1}^{2} \leq\left(\left|\mathbf{w}_{h}\right|_{1}+\left\|r_{h}\right\|_{0}\right) S
$$


where for case i) we define $\tilde{\nu}=\nu$ and $\tilde{\nu}_{s}=\nu$.

Pressure estimate. Consider a nonzero element $\mathbf{v}_{h} \in Y_{h}$. We have

$$
\left(r_{h}, \nabla \cdot \mathbf{v}_{h}\right)=-B_{h}\left(\mathbf{w}_{h}, r_{h} ; \mathbf{v}_{h}, 0\right)+a\left(\mathbf{w}_{h}, \mathbf{v}_{h}\right)-\mathbf{S}_{h}\left(\mathcal{R}_{h}\left(\mathcal{B} \mathbf{v}_{h}\right), \mathbf{c}_{h}\right) .
$$

Remark that $B_{h}\left(\mathbf{w}_{h}, r_{h} ;-\mathbf{v}_{h}, 0\right) \leq S\left|\mathbf{v}_{h}\right|_{1}$. Observe also that

$$
\begin{aligned}
\mathbf{S}_{h}\left(\mathcal{R}_{h}\left(\mathcal{B} \mathbf{v}_{h}\right), \mathbf{c}_{h}\right) & =\left\langle\mathcal{B} \mathbf{v}_{h}, \mathbf{c}_{h}\right\rangle=-\left(\mathbf{u} \cdot \nabla \mathbf{v}_{h}, \mathbf{c}_{h}\right)-\varepsilon \nu\left(\nabla \mathbf{v}_{h}, \nabla \mathbf{c}_{h}\right) \\
& \leq[\mathcal{M}(\mathbf{u})+|\varepsilon-1| \nu]\left|\mathbf{v}_{h}\right|_{1}\left|\mathbf{c}_{h}\right|_{1} .
\end{aligned}
$$

Consequently,

$$
\begin{aligned}
\left(r_{h}, \nabla \cdot \mathbf{v}_{h}\right) & \leq\left\{S+\mathcal{M}(\mathbf{u})\left|\mathbf{w}_{h}\right|_{1}+[\mathcal{M}(\mathbf{u})+|\varepsilon-1| \nu]\left|\mathbf{c}_{h}\right|_{1}\right\}\left|\mathbf{v}_{h}\right|_{1} \leq \\
& \leq C_{1}\left(S+\left|\mathbf{w}_{h}\right|_{1}+\left|\mathbf{c}_{h}\right|_{1}\right)\left|\mathbf{v}_{h}\right|_{1},
\end{aligned}
$$

where $C_{1}=\max \{1, \mathcal{M}(\mathbf{u})+|\varepsilon-1| \nu\}$.

Also, given a nonzero element $\mathbf{z}_{h} \in Z_{h}$,

$$
\begin{aligned}
\left(r_{h}, \nabla \cdot \mathbf{z}_{h}\right) & =-\left\langle\nabla r_{h}, \mathbf{z}_{h}\right\rangle=-\mathbf{S}_{h}\left(\mathcal{R}_{h}\left(\nabla r_{h}\right), \mathbf{z}_{h}\right) \\
& =\mathbf{S}_{h}\left(\mathcal{R}_{h}\left(\mathcal{A} \mathbf{w}_{h}\right), \mathbf{z}_{h}\right)-\mathbf{S}_{h}\left(\mathbf{c}_{h}, \mathbf{z}_{h}\right) \\
& \leq \mathcal{M}_{s}\left[\left|\mathcal{R}_{h}\left(\mathcal{A} \mathbf{w}_{h}\right)\right|_{1}+\left|\mathbf{c}_{h}\right|_{1}\right]\left|\mathbf{z}_{h}\right|_{1} \\
& \leq \mathcal{M}_{s}\left[\nu_{s}^{-1}\left|\mathcal{A} \mathbf{w}_{h}\right|_{-1}+\left|\mathbf{c}_{h}\right|_{1}\right]\left|\mathbf{z}_{h}\right|_{1} \\
& \leq C_{2}\left(\left|\mathbf{w}_{h}\right|_{1}+\left|\mathbf{c}_{h}\right|_{1}\right)\left|\mathbf{z}_{h}\right|_{1}
\end{aligned}
$$

where $C_{2}=\mathcal{M}_{s} \max \left\{\nu_{s}^{-1} \mathcal{M}(\mathbf{u}), 1\right\}$. Then, using Hypothesis 1,

$$
\begin{aligned}
\left(r_{h}, \nabla \cdot\left(\mathbf{z}_{h}+\mathbf{v}_{h}\right)\right) & \leq C_{3}\left(S+\left|\mathbf{w}_{h}\right|_{1}+\left|\mathbf{c}_{h}\right|_{1}\right)\left(\left|\mathbf{v}_{h}\right|_{1}+\left|\mathbf{z}_{h}\right|_{1}\right) \\
& \leq C_{0} C_{3}\left(S+\left|\mathbf{w}_{h}\right|_{1}+\left|\mathbf{c}_{h}\right|_{1}\right)\left|\mathbf{v}_{h}+\mathbf{z}_{h}\right|_{1}
\end{aligned}
$$

where $C_{3}=\max \left\{C_{1}, C_{2}\right\}$. Now, we use the discrete inf-sup condition: There exists a constant $\alpha>0$ such that

$$
\alpha\left\|q_{h}\right\|_{0} \leq \sup _{\mathbf{x}_{h} \in Y_{h}+Z_{h}} \frac{\left(q_{h}, \nabla \cdot \mathbf{x}_{h}\right)}{\left|\mathbf{x}_{h}\right|_{1}}, \quad \forall q_{h} \in M_{h} .
$$

Therefore,

$$
\left\|r_{h}\right\|_{0} \leq C_{4}\left(S+\left|\mathbf{w}_{h}\right|_{1}+\left|\mathbf{c}_{h}\right|_{1}\right)
$$

where $C_{4}=\alpha^{-1} C_{0} C_{3}$.

Conclusion. Combining (18) and (22) and applying Young's inequality yields

$$
\begin{aligned}
\tilde{\nu}\left|\mathbf{w}_{h}\right|_{1}^{2}+\tilde{\nu}_{s}\left|\mathbf{c}_{h}\right|_{1}^{2} \leq & C_{4} S^{2}+\left[\left(1+C_{4}\right)\left|\mathbf{w}_{h}\right|_{1}+C_{4}\left|\mathbf{c}_{h}\right|_{1}\right] S \\
\leq & \frac{1}{2}\left[\left(1+C_{4}\right) \varepsilon_{1}\left|\mathbf{w}_{h}\right|_{1}^{2}+C_{4} \varepsilon_{2}\left|\mathbf{c}_{h}\right|_{1}^{2}\right] \\
& +\left[C_{4}+\frac{1}{2}\left(1+C_{4}\right) \varepsilon_{1}^{-1}+\frac{1}{2} C_{4} \varepsilon_{2}^{-1}\right] S^{2},
\end{aligned}
$$

for any $\varepsilon_{1}>0, \varepsilon_{2}>0$. Let us take $\varepsilon_{1}=\frac{\tilde{\nu}}{1+C_{4}}, \varepsilon_{2}=\frac{\tilde{\nu}_{s}}{C_{4}}$. Then,

$$
\tilde{\nu}\left|\mathbf{w}_{h}\right|_{1}^{2}+\tilde{\nu}_{s}\left|\mathbf{c}_{h}\right|_{1}^{2} \leq C_{5}^{2} S^{2}
$$


where $C_{5}=\left(2 C_{4}+\frac{\left(1+C_{4}\right)^{2}}{\tilde{\nu}}+\frac{C_{4}^{2}}{\tilde{\nu}_{s}}\right)^{1 / 2}$. Thus,

$$
\left|\mathbf{w}_{h}\right|_{1} \leq \frac{C_{5}}{\sqrt{\tilde{\nu}}} S, \quad\left|\mathbf{c}_{h}\right|_{1} \leq \frac{C_{5}}{\sqrt{\tilde{\nu}_{s}}} S
$$

Combining now (23) with (22), we obtain

$$
\left\|r_{h}\right\|_{0} \leq C_{6} S, \quad \text { where } C_{6}=C_{4}+C_{4} C_{5}\left(\frac{1}{\sqrt{\tilde{\nu}}}+\frac{1}{\sqrt{\tilde{\nu}_{s}}}\right) .
$$

From (24) and (25) we finally deduce

$$
S \geq \gamma\left(\left|\mathbf{w}_{h}\right|_{1}+\left\|r_{h}\right\|_{0}+\left|\mathbf{c}_{h}\right|_{1}\right), \quad \text { where } \gamma=\left\{C_{6}+\frac{C_{5}}{\sqrt{\tilde{\nu}}}+\frac{C_{5}}{\sqrt{\tilde{\nu}_{s}}}\right\}^{-1} .
$$

The proof of the second inequality in Definition 1 follows from similar arguments.

The following result closes the equivalence between discrete inf-sup condition and stability of method (6). Thus, the stability analysis of mixed method and method (6) are fully parallel.

Theorem 3. Assume that abstract method (6) is stable for some $\nu_{s}>0$. Assume that Hypothesis 1 and 2 hold. Then, the pairs of spaces $\left\{Y_{h}+Z_{h}, M_{h}\right\}_{h>0}$ satisfy the discrete Brezzi-Babuška condition.

We omit the proof of this result as it again follows from arguments similar to those used in the proof of Theorem 1.

The stability of form $B_{h}$ yields the well-possedness of our method, and allows to derive error estimates, similarly to the standard analysis of mixed methods:

Corollary 1. Under the hypotheses of Theorem 1, problem (6) admits a unique solution $\left(\mathbf{y}_{h}, p_{h}\right) \in Y_{h} \times M_{h}$, that verifies, for some constant $C>0$,

$$
\left|\mathbf{y}_{h}\right|_{1}+\left\|p_{h}\right\|_{0}+\left|\mathbf{z}_{h}\right|_{1} \leq C\|\mathbf{f}\|_{-1}
$$

and

$$
\left|\mathbf{y}-\mathbf{y}_{h}\right|_{1}+\left\|p-p_{h}\right\|_{0}+\left|\mathbf{z}_{h}\right|_{1} \leq C\left[\inf _{\mathbf{v}_{h} \in Y_{h}}\left|\mathbf{y}-\mathbf{v}_{h}\right|_{1}+\inf _{q_{h} \in M_{h}}\left\|p-q_{h}\right\|_{0}\right]
$$

where $\mathbf{z}_{h}=\mathcal{R}_{h}\left(\mathcal{A} \mathbf{y}_{h}+\nabla p_{h}-\mathbf{f}\right)$.

Remark 1. From this result, the "bubble" space $Z_{h}$ appears as a control space for high-frequency components of the residual $\mathcal{A} \mathbf{y}_{h}+\nabla p_{h}-\mathbf{f}$. In fact, (28) shows that the high frequency components of the residual which are representable on $Z_{h}$, via the condensation operator $\mathcal{R}_{h}$, are bounded.

\section{Application to stabilized Methods}

In this section we prove that stabilized methods (10) may be formulated as particular cases of abstract method (6), and then apply the general stability analysis of Section 3.

Our derivation starts from the construction of virtual bubbles developped in Baiocchi et al. [4]. Let us recall the main result of that paper, that we adapt to our context. Consider a Hilbert space $\left(H,(\cdot, \cdot)_{H}\right)$. Given a subset $B$ of $H$ of finite dimension, we define the abstract static condensation operator $\mathcal{R}: H^{\prime} \rightarrow B$ by:

Given $\varphi \in H^{\prime}, \mathcal{R}(\varphi)$ is the only element of $B$ that satisfies

$$
(\mathcal{R}(\varphi), \zeta)_{H}=\langle\varphi, \zeta\rangle, \forall \zeta \in B
$$


Consider also a subspace $W$ of $H^{\prime}$ of finite dimension endowed with an inner product $(\cdot, \cdot)_{W}$. With these ingredients, we may re-write the concept of space of virtual bubbles reproducing an operator on $W$. This is done as follows.

Definition 2. Consider a self-adjoint operator $T$ on $W$. We say that $B$ is a space of virtual bubbles reproducing $T$ on $\left(W,(\cdot, \cdot)_{W}\right)$ with respect to the inner product $(\cdot, \cdot)_{H}$ if

$$
\left(\mathcal{R}\left(\mathbf{w}_{1}\right), \mathcal{R}\left(\mathbf{w}_{2}\right)\right)_{H}=\left(T \mathbf{w}_{2}, \mathbf{w}_{1}\right)_{W}, \quad \forall \mathbf{w}_{1}, \mathbf{w}_{2} \in W
$$

A slight modification of the analysis made in Baiocchi et al. [4], proves the following:

Theorem 4. Let $H$ be an infinite-dimensional Hilbert space. Consider a subset $W$ of $H^{\prime}$ of finite dimension endowed with an inner product $(\cdot, \cdot)_{W}$. Let $B_{0}$ be a finite-dimensional subspace of $H$ satisfying the following property:

$$
\text { If }\langle w, b\rangle=0, \forall b \in B_{0}, \text { for some } w \in W, \text { then } w=0 \text {. }
$$

Then, there exists a constant $\mu_{0}>0$ depending only on $B_{0}$ such that if $0<\tau \leq \mu_{0}$, there exists a finitedimensional space of virtual bubbles $B \subset H$, that reproduces the operator $\tau I$ on $W$ with respect to the inner product $(\cdot, \cdot)_{H}$.

Space $B$ may be constructed as a subspace of $B_{0} \bigoplus N, N$ being any subspace of $H$ of dimension dim $(W)$ such that any function $n \in N$ satisfies

$$
\langle w, n\rangle=0, \quad \forall w \in W, \quad(n, b)_{H}=0, \quad \forall b \in B_{0}
$$

On this base, we may perform the analysis of stabilized methods as particular cases of the abstract method (6):

Proof of Theorem 2.

\section{Step 1: Embedding of stabilized method in abstract method.}

Let us denote $W_{j}^{*}=\left[R_{j}\left(K^{*}\right)\right]^{d}$ for some integer $j \geq 0$ (recall that $K^{*}$ denotes the reference element). By Theorem 4, there exists a constant $\mu^{*}>0$ such that if $0<\tau^{*} \leq \mu^{*}$, there exists a finite-dimensional space of virtual bubbles $B_{j}^{*} \subset\left[H_{0}^{1}\left(K^{*}\right)\right]^{d}$, that reproduces the operator $\tau^{*} I^{*}$ on $W_{j}^{*}$ with respect to the inner product on $\left[H_{0}^{1}\left(K^{*}\right)\right]^{d}$.

Indeed, the elements of $W_{j}^{*}$ are elements of $\left[H^{-1}\left(K^{*}\right)\right]^{d}$ if we identify the $H_{0}^{1}-H^{-1}$ duality with the $L^{2}$ inner product. We consider $W_{j}^{*}$ to be endowed with the $L^{2}$ inner product. Consider a polynomial function $\Phi: K^{*} \mapsto \mathbf{R}$ such that $\Phi=0$ on $\partial K^{*}$, and $\Phi>0$ in $\operatorname{int}\left(K^{*}\right)$. Define the set $B_{0}=\left\{\Phi w^{*}, \mid w^{*} \in W_{j}^{*}\right\}$. Then, $B_{0}$ is a subspace of $\left[H_{0}^{1}\left(K^{*}\right)\right]^{d}$ of dimension $\operatorname{dim}\left(W_{j}^{*}\right)$ satisfying property $(29)$ : Denote by $(\cdot, \cdot)_{*}$ the standard inner product on $\left[L^{2}\left(K^{*}\right)\right]^{d}$. If for some $w^{*} \in W_{j}^{*}$ we have $\left\langle w^{*}, b\right\rangle=\left(w^{*}, b\right)_{*}=0$ for any $b \in B_{0}$, by taking $b=\Phi w^{*}$ we deduce $w^{*}=0$.

Let us now introduce the following elements:

- The bubble finite element space $B_{h}^{(j)} \subset\left[H_{0}^{1}(\Omega)\right]^{d}$ generated by the reference space $B_{j}^{*}$ on triangulation $\mathcal{T}_{h}$.

- The finite element space $W_{h}^{(j)} \subset\left[L^{2}(\Omega)\right]^{d}$ generated by the reference space $W_{j}^{*}$ on triangulation $\mathcal{T}_{h} ;$ i.e.,

$$
W_{h}^{(j)}=\left\{\mathbf{w} \in\left[L^{2}(\Omega)\right]^{d} \mid \mathbf{w}_{\left.\right|_{K}} \in\left[R_{j}(K)\right]^{d}, \forall K \in \mathcal{T}_{h}\right\} .
$$


- The inner forms on $\left[H_{0}^{1}(\Omega)\right]^{d}$,

$$
\begin{aligned}
\mathbf{S}_{h}(\mathbf{w}, \mathbf{v})= & \sum_{K \in \mathcal{T}_{h}} \mathbf{S}_{K}\left(\mathbf{w}_{\left.\right|_{K}}, \mathbf{v}_{\left.\right|_{K}}\right) \text {, where } \\
\mathbf{S}_{K}(\mathbf{w}, \mathbf{v})= & \beta_{K} \int_{K}\left(C_{K} \nabla \mathbf{w}\right): \nabla \mathbf{v} \mathrm{d} x \\
= & \beta_{K} \sum_{j, k, l=1}^{d} \int_{K}\left(C_{K}\right)_{j k} \partial_{l} w_{k} \partial_{l} v_{j} \mathrm{~d} x \text {, with } \beta_{K}=\tau^{*} \tau_{K}^{-1} h_{K}^{2}, \\
& \quad \text { for any } \mathbf{w}=\left(w_{1}, \cdots, w_{d}\right), \mathbf{v}=\left(v_{1}, \cdots, v_{d}\right) \in\left[H^{1}(K)\right]^{d},
\end{aligned}
$$

where $C_{K}$ is the matrix defined as follows: There exists a one-to-one affine mapping $F_{K}$ from the reference element $K^{*}$ into $K$. Its equations are of the form $x=A_{K} x^{*}+b_{K}$, where $A_{K}=\nabla F_{K}$ is a nonsingular $d \times d$ matrix and $b_{K}=F_{K}(0)$ is a vector of $\mathbf{R}^{d}$. We define the matrix $C_{K}$ by $C_{K}=\frac{1}{h_{K}^{2}} A_{K} A_{K}^{t}$.

- The static condensation operator acting on $B_{h}^{(j)}$ associated to $\mathbf{S}_{h}$, that we denote $\mathcal{R}_{h}^{(j)}$.

Then, we have the following representation lemma for the stabilizing terms:

Lemma 1. Assume $\mathbf{f} \in\left[L^{2}(\Omega)\right]^{d}$. Assume $\tau^{*} \leq \mu^{*}$. Then, $\forall \mathbf{w}_{1}, \mathbf{w}_{2} \in W_{h}^{(j)}$,

$$
\sum_{K \in \mathcal{T}_{h}} \tau_{K}\left(\mathbf{w}_{2}-\mathbf{f}, \mathbf{w}_{1}\right)_{K}=\mathbf{S}_{h}\left(\mathcal{R}_{h}^{(j)}\left(\mathbf{w}_{1}\right), \mathcal{R}_{h}^{(j)}\left(\mathbf{w}_{2}-\mathbf{f}_{h}\right)\right)
$$

where $\mathbf{f}_{h}$ is the $L^{2}$ orthogonal projection of $\mathbf{f}$ onto $W_{h}^{(j)}$.

This lemma is proved in the Appendix.

As a consequence, the stabilized method (10) coincides with the abstract method (6) constructed with spaces $Y_{h}=Y_{h}^{(m)}, M_{h}=M_{h}^{(l)}, Z_{h}=B_{h}^{(j)}$ (for a fixed positive parameter $\tau^{*} \leq \mu^{*}$ ), with $j=\max \{2 m-1, l-1\}$, the form $\mathbf{S}_{h}$ given by (32), and with $\mathcal{R}_{h}^{(j)}(\mathbf{f})$ replaced by $\mathcal{R}_{h}^{(j)}\left(\mathbf{f}_{h}\right), \mathbf{f}_{h}$ being the $L^{2}$ orthogonal projection of $\mathbf{f}$ onto $W_{h}^{(j)}$.

Indeed, let us recall that we are assuming that in the stabilizing terms of method (10) the velocity $\mathbf{u}$ is being replaced by some stable interpolate $\mathbf{u}_{h} \in Y_{h}^{(m)}$. Then, for each element $K \in \mathcal{T}_{h}, W_{h}^{(j)}(K)$ contains the set

$$
\left\{\sum_{K \in \mathcal{T}_{h}}\left(\mathbf{u}_{h} \cdot \nabla \mathbf{v}_{h}\right)_{\left.\right|_{K}} 1_{K}, \sum_{K \in \mathcal{T}_{h}}\left(\Delta \mathbf{v}_{h}\right)_{\left.\right|_{K}} 1_{K}, \sum_{K \in \mathcal{T}_{h}}\left(\nabla q_{h}\right)_{\left.\right|_{K}} 1_{K}, \text { for } \mathbf{v}_{h} \in Y_{h}^{(m)}, q_{h} \in M_{h}^{(l)}\right\},
$$

where $1_{K}$ denotes the characteristic function of $K$. Then, it is enough to apply Lemma 1 to obtain the formal embedding.

\section{Step 2: Proof of Hypothesis 1.}

This is based on the general result that follows.

Lemma 2. Assume that the family of triangulations $\left\{\mathcal{T}_{h}\right\}_{h>0}$ is regular. Assume $Y_{h}, Z_{h}$ are finite element subspaces of $\left[H_{0}^{1}(\Omega)\right]^{d}$ affine equivalent to reference spaces $Y^{*}$ and $Z^{*}$, respectively, satisfying $Y^{*} \cap Z^{*}=\{0\}$ and such that $Y^{*}$ contains the constant functions. Then, the pairs of spaces $\left\{Y_{h}, Z_{h}\right\}_{h>0}$ satisfy Hypothesis 1.

This Lemma is proved in the Appendix.

We may now prove that there exists a bubble finite element space $B_{h}^{(j)}$ such that the pairs $\left\{Y_{h}^{(m)}, B_{h}^{(j)}\right\}_{h>0}$ satisfy Hypothesis 1 and the representation formula (34) holds. Indeed, replace the function $\Phi$ that defines space $B_{0}$ by a power $\Phi^{i}$, for some integer $i$ large enough, to ensure $B_{0} \cap\left[R_{m}\left(K^{*}\right)\right]^{d}=\varnothing$. Let $N$ be any subspace 
of $\operatorname{dimension} \operatorname{dim}\left(W_{j}^{*}\right)$ of $\left[H_{0}^{1}\left(K^{*}\right)\right]^{d}$ formed by non-polynomial functions that satisfy property (30). Then, the bubble space $B_{j}^{*}$ given by Theorem 4 satisfies $B_{j}^{*} \cap\left[R_{m}\left(K^{*}\right)\right]^{d}=\varnothing$. Moreover, as $m \geq 1$, then $\left[R_{m}\left(K^{*}\right)\right]^{d}$ contains the constant functions and thus Lemma 2 holds.

Step 3: Proof of Hypothesis 2.

Following the derivation of Lemma 2, we obtain

$$
\mathbf{S}_{h}(\mathbf{w}, \mathbf{w}) \geq \nu_{s h}|\mathbf{w}|_{1}^{2}, \quad\left|\mathbf{S}_{h}(\mathbf{w}, \mathbf{v})\right| \leq M_{s h}|\mathbf{w}|_{1}|\mathbf{v}|_{1}, \quad \forall \mathbf{w}, \mathbf{v} \in\left[H_{0}^{1}(\Omega)\right]^{d},
$$

where $\nu_{s h}=\Lambda \min _{K \in \mathcal{T}_{h}}\left\{\beta_{K}\right\}, M_{s h}=M \max _{K \in \mathcal{T}_{h}}\left\{\beta_{K}\right\}$, the constants $\Lambda$ and $M$ being given by (81). Observe that

$$
\alpha_{2}^{-1} \tau^{*} \leq \beta_{K} \leq \alpha_{1}^{-1} \tau^{*}, \quad \forall K \in \mathcal{T}_{h},
$$

where $\alpha_{1}$ (assuming $h_{K} \leq 1$ ) and $\alpha_{2}$ are given by

$$
\alpha_{1}=A \min \left\{\frac{1}{\nu}, \frac{P}{\|\mathbf{u}\|_{0, p}}\right\}, \quad \alpha_{2}=\frac{A}{\nu} .
$$

Then, $\nu_{s h} \geq \nu_{s}, M_{s h} \geq M_{s}$ uniformly in $h$, with

$$
\nu_{s}=\alpha_{2}^{-1} \tau^{*} \Lambda, \quad M_{s}=\alpha_{1}^{-1} \tau^{*} M .
$$

\section{Step 4: Discrete inf-sup condition.}

This will be based upon the following:

Lemma 3. Assume that for each $h>0$ there exists a subspace $Z_{h} \subset\left[H_{0}^{1}(\Omega)\right]^{d}$ and a coercive bilinear form $\hat{\mathbf{S}}_{h}$ on $\left[H_{0}^{1}(\Omega)\right]^{d}$ such that $Z_{h} \cap Y_{h}^{(m)}=\{0\}$ and

$$
\sum_{K \in \mathcal{T}_{h}} h_{K}^{2}\left\|\nabla q_{h}\right\|_{0, K}^{2} \leq \hat{C} \hat{\mathbf{S}}_{h}\left(\hat{\mathcal{R}}\left(\nabla q_{h}\right), \hat{\mathcal{R}}\left(\nabla q_{h}\right)\right) \forall q_{h} \in M_{h}^{(l)},
$$

for some constant $\hat{C}>0$, where $\hat{\mathcal{R}}$ denotes the static condensation operator on $Z_{h}$ with respect to the form $\hat{\mathbf{S}}_{h}$. Assume that the family of forms $\left\{\hat{\mathbf{S}}_{h}\right\} h>0$ is uniformly coercive on $\left[H_{0}^{1}(\Omega)\right]^{d}$. Then, under condition (13), the pairs of spaces $\left\{Y_{h}^{(m)}+Z_{h}, M_{h}^{(l)}\right\}_{h>0}$ satisfy the discrete inf-sup condition.

Proof of Lemma 3. Under condition (13), it is proved in Franca et al. [16] - using the trick of Verfürth [28] that there exist two constants $C_{1}>0, C_{2}>0$ such that $\forall q_{h} \in M_{h}^{(l)}$,

$$
\sup _{\mathbf{v} \in Y_{h}^{(m)}-\{0\}} \frac{\left(\nabla \cdot \mathbf{v}_{h}, q_{h}\right)}{\left|\mathbf{v}_{h}\right|_{1}} \geq C_{1}\left\|q_{h}\right\|_{0}-C_{2}\left(\sum_{K \in \mathcal{T}_{h}} h_{K}^{2}\left\|\nabla q_{h}\right\|_{0, K}^{2}\right)^{1 / 2} .
$$

Then,

$$
\sup _{\mathbf{v} \in Y_{h}^{(m)}-\{0\}} \frac{\left(\nabla \cdot \mathbf{v}_{h}, q_{h}\right)}{\left|\mathbf{v}_{h}\right|_{1}} \geq C_{1}\left\|q_{h}\right\|_{0}-C_{3}\left[\hat{\mathbf{S}}_{h}\left(\hat{\mathcal{R}}\left(\nabla q_{h}\right), \hat{\mathcal{R}}\left(\nabla q_{h}\right)\right)\right]^{1 / 2}
$$

where $C_{3}=\hat{C} C_{2}$. Consider a nonzero element $\tilde{\mathbf{v}}_{h} \in Y_{h}^{(m)}$, such that

$$
\left|\tilde{\mathbf{v}}_{h}\right|_{1}=1, \quad \frac{\left(\nabla \cdot \tilde{\mathbf{v}}_{h}, q_{h}\right)}{\left|\tilde{\mathbf{v}}_{h}\right|_{1}}=\sup _{\mathbf{v} \in Y_{h}^{(m)}-\{0\}} \frac{\left(\nabla \cdot \mathbf{v}_{h}, q_{h}\right)}{\left|\mathbf{v}_{h}\right|_{1}} .
$$


Denote $\mathbf{z}_{h}=\hat{\mathcal{R}}\left(\nabla q_{h}\right)$. If $\mathbf{z}_{h}=0$, then

$$
\left(\nabla \cdot \tilde{\mathbf{v}}_{h}, q_{h}\right) \geq C_{1}\left\|q_{h}\right\|_{0}
$$

If $\mathbf{z}_{h} \neq 0$, denote $\tilde{\mathbf{z}}_{h}=\mathbf{z}_{h} /\left|\mathbf{z}_{h}\right|_{1}$. Given a constant $C_{4} \geq 0$, from (38) and (36) we obtain

$$
\begin{aligned}
\left(\nabla \cdot\left(\tilde{\mathbf{v}}_{h}-C_{4} \tilde{\mathbf{z}}_{h}\right), q_{h}\right) & \geq C_{1}\left\|q_{h}\right\|_{0}-C_{3}\left[\hat{\mathbf{S}}_{h}\left(\mathbf{z}_{h}, \mathbf{z}_{h}\right)\right]^{1 / 2}+C_{4} \hat{\mathbf{S}}_{h}\left(\mathbf{z}_{h}, \tilde{\mathbf{z}}_{h}\right) \\
& \geq C_{1}\left\|q_{h}\right\|_{0}+\left\{C_{4}\left[\hat{\mathbf{S}}_{h}\left(\tilde{\mathbf{z}}_{h}, \tilde{\mathbf{z}}_{h}\right)\right]^{1 / 2}-C_{3}\right\}\left|\mathbf{z}_{h}\right|_{1}\left[\hat{\mathbf{S}}_{h}\left(\tilde{\mathbf{z}}_{h}, \tilde{\mathbf{z}}_{h}\right)\right]^{1 / 2} \\
& \geq C_{1}\left\|q_{h}\right\|_{0}+\left(C_{4} \hat{\nu}^{1 / 2}-C_{3}\right)\left|\mathbf{z}_{h}\right|_{1}\left[\hat{\mathbf{S}}_{h}\left(\tilde{\mathbf{z}}_{h}, \tilde{\mathbf{z}}_{h}\right)\right]^{1 / 2},
\end{aligned}
$$

where $\hat{\nu}$ is the uniform coerciveness constant of the forms $\hat{\mathbf{S}}_{h}$. Let us take $C_{4}=\hat{\nu}^{-1 / 2} C_{3}$. Observe that $\tilde{\mathbf{v}}_{h}-C_{4} \tilde{\mathbf{z}}_{h} \neq 0$ as $Y_{h}^{(m)} \cap Z_{h}=\{0\}$. Define $\tilde{\mathbf{x}}_{h}=\frac{\tilde{\mathbf{v}}_{h}-C_{4} \tilde{\mathbf{z}}_{h}}{\left|\tilde{\mathbf{v}}_{h}-C_{4} \tilde{\mathbf{z}}_{h}\right|_{1}} \in Y_{h}^{(m)}+Z_{h}$. Then,

$$
\left(\nabla \cdot \tilde{\mathbf{x}}_{h}, q_{h}\right) \geq \frac{C_{1}}{1+C_{4}}\left\|q_{h}\right\|_{0}
$$

This completes the proof of Lemma 3.

In our case, this result holds with $Z_{h}=B_{h}^{(j)}$. Indeed, on one hand $B_{h}^{(j)} \cap Y_{h}^{(m)}=\varnothing$. On the other hand, let us take $\mathbf{w}_{1}=\mathbf{w}_{2}=\nabla q_{h}, \mathbf{f}=0$ in the representation formula (34). Then,

$$
\sum_{K \in \mathcal{T}_{h}} \tau_{K}\left\|\nabla q_{h}\right\|_{0, K}^{2}=\mathbf{S}_{h}\left(\mathcal{R}_{h}^{(j)}\left(\nabla q_{h}\right), \mathcal{R}_{h}^{(j)}\left(\nabla q_{h}\right)\right)
$$

and (36) follows because the coefficients $\tau_{K}$ are of order $h_{K}^{2}: \alpha_{1} h_{K}^{2} \leq \tau_{K} \leq \alpha_{2} h_{K}^{2}$. Finally, by Step 3 the forms $\mathbf{S}_{h}$ are uniformly coercive.

\section{Step 5: Conclusion.}

We now apply Theorem 1:

- SUPG method corresponds to $m=l=1$, for any $\varepsilon$. In this case, $Y_{h}^{(m)}$ and $B_{h}^{(j)}$ are $H_{0}^{1}$-orthogonal. Then, from Theorem 1, it is stable for any $A>0$.

- GaLS method corresponds to $\varepsilon=1$. Then, from Theorem 1 , it is also stable for any $A>0$.

- In the remaining cases, $Y_{h}^{(m)}$ and $B_{h}^{(j)}$ are not necessarily $H_{0}^{1}$-orthogonal, and $\varepsilon \neq 1$. Let us assume

$$
A \leq A_{0}\left(\frac{2}{\varepsilon-1}\right)^{2}, \text { with } A_{0}=\Lambda \mu^{*}
$$

Take $\tau^{*}$ in the closed interval $\left[A \Lambda^{-1}\left(\frac{\varepsilon-1}{2}\right)^{2}, \mu^{*}\right]$. As $\tau^{*} \leq \mu^{*}$, then all the preceeding analysis applies. Also, $\nu_{s}=A^{-1} \tau^{*} \Lambda \nu \geq\left(\frac{\varepsilon-1}{2}\right)^{2} \nu$. Then, from Theorem 1, the general stabilized method (10) is stable. AdS and generalized SUPG methods respectively correspond to $\varepsilon=-1$ and $\varepsilon=0$ and therefore they are respectively stable if $A \leq \Lambda \mu^{*}$ and $A \leq 4 \Lambda \mu^{*}$.

This completes the proof of Theorem 2 .

\section{Remark 2. Stability of AdS method.}

The stability of AdS method may be proved without using the uniform separation property. Indeed, in Baiocchi et al. [4] it is proved that there exists a bubble subspace $B_{h}$ of $\left[H_{0}^{1}(\Omega)\right]^{d}$ (not necessarily a finite 
element space) such that for any $\left(\mathbf{v}_{h}, q_{h}\right),\left(\mathbf{w}_{h}, r_{h}\right) \in Y_{h}^{(m)} \times M_{h}^{(l)}$ we have

$$
\sum_{K \in \mathcal{T}_{h}} \tau_{K}\left(\mathcal{B} \mathbf{v}_{h}+\nabla q_{h}, \mathcal{A} \mathbf{w}_{h}+\nabla r_{h}-\mathbf{f}\right)_{K}=a\left(\mathcal{R}_{h}\left(\mathcal{B} \mathbf{v}_{h}+\nabla q_{h}\right), \mathcal{R}_{h}\left(\mathcal{A} \mathbf{w}_{h}+\nabla r_{h}-\mathbf{f}_{h}\right)\right),
$$

where $\mathcal{R}_{h}$ is the static condensation operator on $B_{h}$ with respect to the bilinear form $a(\cdot, \cdot)$. This occurs whenever $\tau_{K} \leq \mu_{K}$ for some positive $\mu_{K}$.

As it is proved in Baiocchi et al. [4], this implies that a pair $\left(\mathbf{y}_{h}, r_{h}\right) \in Y_{h}^{(m)} \times M_{h}^{(l)}$ is a solution of AdS method if and only if the pair $\left(\mathbf{y}_{h}+\mathbf{b}_{h}, p_{h}\right) \in\left(Y_{h}^{(m)}+B_{h}\right) \times M_{h}^{(l)}$, where $\mathbf{b}_{h}=\mathcal{R}_{h}\left(\mathbf{f}-\left(\mathcal{A} \mathbf{y}_{h}+\nabla p_{h}\right)\right)$, is a solution of the mixed method constructed with spaces $Y_{h}=Y_{h}^{(m)}+B_{h}$ and $M_{h}=M_{h}^{(l)}$ :

$$
B\left(\mathbf{y}_{h}+\mathbf{b}_{h}, p_{h} ; \mathbf{v}_{h}, q_{h}\right)=\left\langle\mathbf{f}, \mathbf{v}_{h}\right\rangle, \quad \forall\left(\mathbf{v}_{h}, q_{h}\right) \in\left(Y_{h}^{(m)}+B_{h}\right) \times M_{h}^{(l)} .
$$

Thus, to apply the standard analysis of mixed methods to AdS method it is enough to prove that the family of pairs of spaces $\left\{Y_{h}^{(m)}+B_{h}, M_{h}^{(l)}\right\}_{h>0}$ satisfy the discrete inf-sup condition. This may be proved by Lemma 3 starting from (41), once we prove that the upper bounds $\mu_{K}$ for the stabilizing coefficients are of order $h_{K}^{2}$.

Notice that equation (42) provides two control equations for the large and small scale components of $\nabla p_{h}$. Indeed, (42) is equivalent to

$$
\begin{array}{ll}
\left(p_{h}, \nabla \cdot \mathbf{v}_{h}\right)=a\left(\mathbf{y}_{h}+\mathbf{b}_{h}, \mathbf{v}_{h}\right)-\left\langle\mathbf{f}, \mathbf{v}_{h}\right\rangle, & \forall \mathbf{v}_{h} \in Y_{h} ; \\
\left(p_{h}, \nabla \cdot \mathbf{z}_{h}\right)=a\left(\mathbf{y}_{h}+\mathbf{b}_{h}, \mathbf{z}_{h}\right)-\left\langle\mathbf{f}, \mathbf{z}_{h}\right\rangle, \quad \forall \mathbf{z}_{h} \in Z_{h} .
\end{array}
$$

In the case of stabilized methods other than AdS, we no longer have $\mathcal{B}=\mathcal{A}^{*}$ in (41). Then, we cannot write the method under the structure (42). In this case, the control equations for $\nabla p_{h}$ are (19) and (20).

\section{Remark 3. Computability of stability bounds.}

In Baiocchi et al. [4], Section 3.1, a general technique for estimating $\mu^{*}$ is derived. The parameter $\mu^{*}$ depends only on the reference element $B_{j}^{*}$. It must be computed once for each actual space $W_{j}^{*}$ associated to a pair $\left(Y_{h}^{(m)}, M_{h}^{(l)}\right)$. In the case of two-dimensional triangular elements, for instance, for $m=l=1$, this technique yields the estimate $\mu^{*}=1 / 320$. If $m=l=2, \mu^{*}=3 / 5120$.

Also, the constant $\Lambda$ may be computed from the aspect ratio of the family of triangulations $\left\{\mathcal{T}_{h}\right\}_{h>0}$. Recall that $\Lambda=C_{1}^{2}, C_{1}$ being the constant appearing in (80). From Ciarlet [13], this constant is

$$
C_{1}=\frac{1}{2 h^{*} \sigma},
$$

where $h^{*}$ is the diameter of the reference element $K^{*}$ and $\sigma$ is the aspect ratio of the family,

$$
\sigma=\sup _{h>0} \max _{K \in \mathcal{T}_{h}} \frac{h_{K}}{\rho_{K}}
$$

$\rho_{K}$ denoting the internal diameter of element $K$. This technique to estimate the stability bounds simplifies the standard one, that requires computing the best constant in the inverse inequality (12). This simplification is particularly clear if we observe that $\sigma$ may be preset "a priori" if the triangulations are constructed in order to have

$$
\frac{h_{K}}{\rho_{K}} \leq \sigma, \quad \forall K \in \mathcal{T}_{h}, \forall h>0 .
$$

\section{Remark 4. Error estimates.}

Let us finally make some comments about the obtention of error estimates. One may experiment some concern by the fact that to represent the stabilized method as an abstract method, in the stabilizing terms 
the second member $\mathbf{f}$ is replaced by its $L^{2}$ interpolate on $W_{h}^{(j)}, \mathbf{f}_{h}$. However, we still obtain error estimates of optimal order. This is proved for Navier-Stokes equations in [11]. This proof may readily be adapted to Oseen equations.

\section{Application to stabilized Spectral Element method}

In Gervasio and Saleri [19], a stabilized spectral element (SSE) method for solving the unsteady Navier-Stokes equations is derived. We shall apply here our analysis to the approximation of the Oseen equations by such method. Our main contribution is to prove that the stability of the discretization is due to an underlying discrete inf-sup condition. This allows to obtain $L^{2}$ estimates for the pressure. Oseen equations are here considered as a model problem for the linear problems that appear after time discretization of Navier-Stokes equations.

Let us start by describing the discretization of Oseen equations by the SSE method. Assume $\Omega$ to be polygonal. Consider a partition $\mathcal{T}_{h}$ of $\Omega$ in parallelograms $(d=2)$ or parallelepipeds $(d=3)$, where $h$ still denotes the largest diameter of the elements of $\mathcal{T}_{h}$.

Consider an integer number $N \geq 1$. Denote by $\left\{\xi_{i}\right\}_{i=1}^{N+1}$ and by $\left\{\omega_{i}\right\}_{i=1}^{N+1}$ the nodes and weights of the GaussLobatto Legendre quadrature formulas defined on $(-1,1)$. Assume, for instance, $d=3$. For $\hat{u}_{N}, \hat{v}_{N} \in Q_{N}\left(K^{*}\right)$ $\left(K^{*}=(-1,1)^{d}\right.$ being the reference element), we define the discrete inner product,

$$
\left(\hat{u}_{N}, \hat{v}_{N}\right)_{N, K^{*}}=\sum_{i, j, k=1}^{N+1} \omega_{i} \omega_{j} \omega_{k} \hat{u}_{N}\left(\xi_{i}, \xi_{j}, \xi_{k}\right) \hat{v}_{N}\left(\xi_{i}, \xi_{j}, \xi_{k}\right) ;
$$

while for $u_{N}, v_{N} \in Q_{N}(K)$ we set

$$
\left(u_{h}, v_{h}\right)_{N, K}=\sum_{i, j, k=1}^{N+1} \omega_{i} \omega_{j} \omega_{k}\left|\operatorname{det} A_{K}\right| u_{N}\left(P_{i j k}^{(K)}\right) v_{N}\left(P_{i j k}^{(K)}\right) ;
$$

where $P_{i j k}^{(K)}=F_{K}\left(\xi_{i}, \xi_{j}, \xi_{k}\right) ; i, j, k=1, \cdots, N+1, \forall K \in \mathcal{T}_{h}$.

Let us define the space

$$
W_{\mathcal{H}}=\left\{v \in L^{2}(\Omega) \mid v_{\left.\right|_{K}} \in Q_{N}(K), \forall K \in \mathcal{T}_{h}\right\},
$$

where $\mathcal{H}=\left(h, N^{-1}\right)$ is our actual discretization parameter.

Given $u_{\mathcal{H}}, v_{\mathcal{H}} \in W_{\mathcal{H}}$ we set

$$
\left(u_{\mathcal{H}}, v_{\mathcal{H}}\right)_{\mathcal{H}}=\sum_{K \in \mathcal{T}_{h}}\left(u_{\mathcal{H}_{\mid}}, v_{\mathcal{H}_{\mid K}}\right)_{N, K} ; \quad\left\|u_{\mathcal{H}}\right\|_{\mathcal{H}}=\left(u_{\mathcal{H}}, u_{\mathcal{H}}\right)_{\mathcal{H}}^{1 / 2}
$$

We respectively define the discrete inner products $(\cdot, \cdot)_{N, K^{*}},(\cdot, \cdot)_{N, K}$ and $(\cdot, \cdot)_{\mathcal{H}}$ on a similar manner for vector functions of $\left[Q_{N}\left(K^{*}\right)\right]^{d},\left[Q_{N}(K)\right]^{d}$, and $\left[W_{\mathcal{H}}\right]^{d}$.

We also define the spectral element spaces

$$
Y_{\mathcal{H}}=V_{\mathcal{H}}^{d} \cap\left[H_{0}^{1}(\Omega)\right]^{d}, \quad M_{\mathcal{H}}=V_{\mathcal{H}} \cap L_{0}^{2}(\Omega),
$$

where $V_{\mathcal{H}}$ is defined by

$$
V_{\mathcal{H}}=\left\{v \in C^{0}(\bar{\Omega}) \mid v_{\left.\right|_{K}} \in Q_{N}(K), \forall K \in \mathcal{T}_{h}\right\} .
$$

We shall consider the following SSE approximation of Oseen equations (1):

$$
\left\{\begin{array}{l}
\text { Obtain }\left(\mathbf{y}_{\mathcal{H}}, p_{\mathcal{H}}\right) \in Y_{\mathcal{H}} \times M_{\mathcal{H}} \text { such that } \\
B_{\mathcal{H}}\left(\mathbf{y}_{\mathcal{H}}, p_{\mathcal{H}} ; \mathbf{v}_{\mathcal{H}}, q_{\mathcal{H}}\right)=F_{\mathcal{H}}\left(\mathbf{v}_{\mathcal{H}}, q_{\mathcal{H}}\right), \quad \forall\left(\mathbf{v}_{\mathcal{H}}, q_{\mathcal{H}}\right) \in Y_{\mathcal{H}} \times M_{\mathcal{H}}
\end{array}\right.
$$


where

$$
\begin{aligned}
B_{\mathcal{H}}\left(\mathbf{w}_{\mathcal{H}}, r_{\mathcal{H}} ; \mathbf{v}_{\mathcal{H}}, q_{\mathcal{H}}\right) & =B\left(\mathbf{w}_{\mathcal{H}}, r_{\mathcal{H}} ; \mathbf{v}_{\mathcal{H}}, q_{\mathcal{H}}\right)-\sum_{K \in \mathcal{T}_{h}} \tau_{K}\left(\mathcal{B}_{\mathbf{v}_{\mathcal{H}}}+\nabla q_{\mathcal{H}} ; \mathcal{A}_{\mathcal{H}}+\nabla r_{\mathcal{H}}\right)_{N, K} ; \\
F_{\mathcal{H}}\left(\mathbf{v}_{\mathcal{H}}, q_{\mathcal{H}}\right) & =\left\langle\mathbf{f}, \mathbf{v}_{\mathcal{H}}\right\rangle-\sum_{K \in \mathcal{T}_{h}} \tau_{K}\left(\mathcal{B}_{\mathbf{v}_{\mathcal{H}}}+\nabla q_{\mathcal{H}}, \mathbf{f}\right)_{N, K}
\end{aligned}
$$

The essential difference between SSE method and stabilized method (10) is that the $L^{2}$ inner products $(\cdot, \cdot)_{K}$ that appear in (10) in the stabilizing terms are here replaced by the discrete inner products $(\cdot, \cdot)_{N, K}$. In Gervasio and Saleri [19], the discrete inner product $(\cdot, \cdot)_{\mathcal{H}}$ is also used to approximate the integral terms appearing in form $B$. Here, for simplicity we prefer to consider the above discretization. However, we may extend our analysis to the actual discretization considered in that paper if the pressures are approximated by piecewise polynomials of degree at most $N-1$ (see Rem. 5).

In Gervasio and Saleri [19], the stabilizing coefficients $\tau_{K}$ are still given by (11), with

$$
P=2 \frac{N^{2}}{m}, A=\frac{m}{4 N^{4}}, \quad \text { for some } m>0 .
$$

The parameter $m$ is determined in that paper in order to obtain uniform-in-time stability of the linear problems that arise after time discretization. We shall simply assume that the stabilizing coefficients $\tau_{K}$ are given by (11).

Our analysis allows to state the following result:

Theorem 5. Assume the triangulations $\left\{\mathcal{I}_{h}\right\}_{h>0}$ are regular. Then, the SSE method (43) is stable for any $A>0$ if $\varepsilon=1$, and for $0<A<\left(\frac{2}{1-\varepsilon}\right)^{2} \hat{A}_{0}$ if $\varepsilon \neq 1$, where $\hat{A}_{0}$ is a computable positive constant.

As a consequence, if $\mathbf{f} \in\left[C^{0}(\Omega)\right]^{d}$, problem (43) admits a unique solution that satisfies

$$
\left|\mathbf{y}_{\mathcal{H}}\right|_{1}+\left\|p_{\mathcal{H}}\right\|_{0} \leq C\|\mathbf{f}\|_{C^{0}}
$$

for some constant $C>0$ independent of $\mathcal{H}$.

Proof. We proceed as in the proof of Theorem 2.

Step 1: Embedding of SSE method in abstract method.

Let us define the local interpolation operator $I_{N}^{K}: C^{0}(K) \rightarrow Q_{N}(K)$ by

$$
\left(I_{N}^{K} w\right)\left(P_{i j k}^{(K)}\right)=w\left(P_{i j k}^{(K)}\right), i, j, k=1, \cdots, N+1 .
$$

Consider the space of piecewise continuous functions on $\mathcal{T}_{h}$,

$$
C_{p, h}(\Omega)=\left\{v \in L^{2}(\Omega) \mid v_{\left.\right|_{K}} \in C^{0}(K), \forall K \in \mathcal{T}_{h}\right\} ;
$$

and define the global interpolation operator $I_{\mathcal{H}}: C_{p, h}(\Omega) \rightarrow W_{\mathcal{H}}$ by

$$
\left(I_{\mathcal{H}} w\right)_{\left.\right|_{K}}=I_{N}^{K}\left(w_{\left.\right|_{K}}\right), \forall K \in \mathcal{T}_{h} .
$$

Observe that $C^{0}(\bar{\Omega}) \subset C_{p, h}(\Omega)$ and that $I_{\mathcal{H}} w \in V_{\mathcal{H}}$ if $w \in C^{0}(\bar{\Omega})$. 
The following representation formula holds:

Lemma 4. There exists a finite-dimensional bubble finite element space $Z_{\mathcal{H}} \subset\left[H_{0}^{1}(\Omega)\right]^{d}$ such that

$$
\mathbf{S}_{h}\left(\mathcal{R}_{\mathcal{H}}\left(I_{\mathcal{H}} \mathbf{v}_{1}\right), \mathcal{R}_{\mathcal{H}}\left(I_{\mathcal{H}} \mathbf{v}_{2}\right)\right)=\sum_{K \in \mathcal{T}_{h}} \tau_{K}\left(\mathbf{v}_{1}, \mathbf{v}_{2}\right)_{N, K}, \quad \forall \mathbf{v}_{1}, \mathbf{v}_{2} \in\left[C_{p, h}(\Omega)\right]^{d}
$$

where $\mathbf{S}_{h}$ is the bilinear form defined by (32).

This lemma is proved in the Appendix.

As a consequence, for all $\mathbf{w}_{\mathcal{H}}, \mathbf{v}_{\mathcal{H}} \in Y_{\mathcal{H}} ; r_{\mathcal{H}}, q_{\mathcal{H}} \in M_{\mathcal{H}}$,

$$
\begin{aligned}
B_{\mathcal{H}}\left(\mathbf{w}_{\mathcal{H}}, r_{\mathcal{H}} ; \mathbf{v}_{\mathcal{H}}, q_{\mathcal{H}}\right)= & B\left(\mathbf{w}_{\mathcal{H}}, r_{\mathcal{H}} ; \mathbf{v}_{\mathcal{H}}, q_{\mathcal{H}}\right) \\
& -\mathbf{S}_{h}\left(\mathcal{R}_{\mathcal{H}}\left(I_{\mathcal{H}}\left(\mathcal{B} \mathbf{v}_{\mathcal{H}}+\nabla q_{\mathcal{H}}\right)\right), \mathcal{R}_{\mathcal{H}}\left(I_{\mathcal{H}}\left(\mathcal{A} \mathbf{w}_{\mathcal{H}}+\nabla r_{\mathcal{H}}\right)\right)\right) ; \\
F_{\mathcal{H}}\left(\mathbf{v}_{\mathcal{H}}, q_{\mathcal{H}}\right)= & \left\langle\mathbf{f}, \mathbf{v}_{\mathcal{H}}\right\rangle-\mathbf{S}_{h}\left(\mathcal{R}_{\mathcal{H}}\left(I_{\mathcal{H}}\left(\mathcal{B} \mathbf{v}_{\mathcal{H}}+\nabla q_{\mathcal{H}}\right)\right), \mathcal{R}_{\mathcal{H}}\left(I_{\mathcal{H}} \mathbf{f}\right)\right) .
\end{aligned}
$$

This occurs because $\mathbf{f} \in\left[C^{0}(\Omega)\right]^{d}$ and $\mathcal{B} \mathbf{v}_{\mathcal{H}}+\nabla q_{\mathcal{H}}, \mathcal{A w}_{\mathcal{H}}+\nabla r_{\mathcal{H}} \in\left[C_{p, h}(\Omega)\right]^{d}$.

Steps 2 and 3: Proof of Hypotheses 1 and 2.

Hypotheses 1 and 2 have respectively been proved in the Steps 3 and 4 of the proof of Theorem 2 .

Step 4: Discrete inf-sup condition.

Observe that if $r_{\mathcal{H}} \in M_{\mathcal{H}}$, then $I_{\mathcal{H}}\left(\nabla r_{\mathcal{H}}\right)=\nabla r_{\mathcal{H}}$, because $I_{N}^{K}\left(q_{N}\right)=q_{N}, \forall q_{N} \in Q_{N}(K)$. Then, by (45),

$$
\mathbf{S}_{h}\left(\mathcal{R}_{\mathcal{H}}\left(\nabla r_{\mathcal{H}}\right), \mathcal{R}_{\mathcal{H}}\left(\nabla r_{\mathcal{H}}\right)\right)=\sum_{K \in \mathcal{T}_{h}} \tau_{K}\left(\nabla r_{\mathcal{H}}, \nabla r_{\mathcal{H}}\right)_{N, K}, \quad \forall r_{\mathcal{H}} \in M_{\mathcal{H}}
$$

By Bernardi and Maday [5],

$$
\left\|q_{N}\right\|_{0, K} \leq\left\|q_{N}\right\|_{N, K} \leq 3\left\|q_{N}\right\|_{0, K}, \quad \forall q_{N} \in Q_{N}(K)
$$

These estimates are obtained by affine transportation of similar estimates obtained in the reference element. As the coefficients $\tau_{K}$ are of order $h_{K}^{2}$, then there exists a constant $C>0$ such that

$$
\sum_{K \in \mathcal{T}_{h}} h_{K}^{2}\left\|\nabla r_{\mathcal{H}}\right\|_{0, K}^{2} \leq C \mathbf{S}_{h}\left(\mathcal{R}_{\mathcal{H}}\left(\nabla r_{\mathcal{H}}\right), \mathcal{R}_{\mathcal{H}}\left(\nabla r_{\mathcal{H}}\right)\right)
$$

Then, by Lemma 3, the pairs of spaces $\left\{Y_{\mathcal{H}}+Z_{\mathcal{H}}, M_{\mathcal{H}}\right\}_{\mathcal{H}>0}$ satisfy the discrete inf-sup condition.

\section{Step 5: Conclusion.}

Following the proof of Theorem 1 , we prove that if $\nu_{s} \geq \frac{(1-\varepsilon)^{2}}{4} \nu$ when $\varepsilon \neq 1$, or $\nu_{s}>0$ when $\varepsilon=1$, then the form $B_{\mathcal{H}}$ is stable. Then, problem (43) admits a unique solution that satisfies, for some constant $C>0$,

$$
\left|\mathbf{y}_{\mathcal{H}}\right|_{1}+\left\|p_{\mathcal{H}}\right\|_{0}+\left|\mathbf{z}_{\mathcal{H}}\right|_{1} \leq C\left(\|\mathbf{f}\|_{-1}+\left|\mathcal{R}_{\mathcal{H}}\left(I_{\mathcal{H}} \mathbf{f}\right)\right|_{1}\right)
$$

where $\mathbf{z}_{\mathcal{H}}=\mathcal{R}_{\mathcal{H}}\left(I_{\mathcal{H}}\left(\mathcal{A y}_{\mathcal{H}}\right)+\nabla p_{\mathcal{H}}\right)$. As $\mathbf{f} \in\left[C^{0}(\Omega)\right]^{d}$, using (47),

$$
\left|\mathcal{R}_{\mathcal{H}}\left(I_{\mathcal{H}} \mathbf{f}\right)\right|_{1} \leq \nu_{s}^{-1}\left\|I_{\mathcal{H}} \mathbf{f}\right\|_{0} \leq \nu_{s}^{-1}\left\|I_{\mathcal{H}} \mathbf{f}\right\|_{\mathcal{H}}=\nu_{s}^{-1}\|\mathbf{f}\|_{\mathcal{H}} \leq C\|\mathbf{f}\|_{C^{0}}
$$

Thus, estimate (44) follows.

The remaining of the proof is similar to the conclusion of the proof of Theorem 2 . 
Remark 5. A slight modification of the above argument allows to prove an underlying inf-sup condition and thus the stability for a stabilized full spectral element discretization of Oseen equations.

Indeed, let us replace the pressure space $M_{\mathcal{H}}$ by $M_{\mathcal{H}^{\prime}}$, with $\mathcal{H}^{\prime}=\left(h,(N-1)^{-1}\right)$, for $N \geq 2$; i.e., we consider pressures of degree at most $N-1$ elementwise. We consider the following discrete problem:

$$
\left\{\begin{array}{l}
\text { Obtain }\left(\mathbf{y}_{\mathcal{H}}, p_{\mathcal{H}^{\prime}}\right) \in Y_{\mathcal{H}} \times M_{\mathcal{H}^{\prime}} \text { such that } \\
B_{\mathcal{H}}^{\prime}\left(\mathbf{y}_{\mathcal{H}}, p_{\mathcal{H}^{\prime}} ; \mathbf{v}_{\mathcal{H}}, q_{\mathcal{H}^{\prime}}\right)=F_{\mathcal{H}}^{\prime}\left(\mathbf{v}_{\mathcal{H}}, q_{\mathcal{H}^{\prime}}\right), \quad \forall\left(\mathbf{v}_{\mathcal{H}}, q_{\mathcal{H}^{\prime}}\right) \in Y_{\mathcal{H}} \times M_{\mathcal{H}^{\prime}}
\end{array}\right.
$$

where

$$
\begin{aligned}
B_{\mathcal{H}}^{\prime}\left(\mathbf{w}_{\mathcal{H}}, r_{\mathcal{H}^{\prime}} ; \mathbf{v}_{\mathcal{H}}, q_{\mathcal{H}^{\prime}}\right)= & \frac{1}{2}\left[\left(\mathbf{u} \cdot \nabla \mathbf{w}_{\mathcal{H}}, \mathbf{v}_{\mathcal{H}}\right)_{\mathcal{H}}-\left(\mathbf{u} \cdot \nabla \mathbf{v}_{\mathcal{H}}, \mathbf{w}_{\mathcal{H}}\right)_{\mathcal{H}}\right]+\nu\left(\nabla \mathbf{w}_{\mathcal{H}}, \nabla \mathbf{v}_{\mathcal{H}}\right)_{\mathcal{H}} \\
& -\left(\nabla \cdot \mathbf{w}_{\mathcal{H}}, q_{\mathcal{H}^{\prime}}\right)_{\mathcal{H}}-\left(r_{\mathcal{H}^{\prime}}, \nabla \cdot \mathbf{v}_{\mathcal{H}}\right)_{\mathcal{H}} \\
& -\sum_{K \in \mathcal{T}_{h}} \tau_{K}\left(\mathcal{B} \mathbf{v}_{\mathcal{H}}+\nabla q_{\mathcal{H}^{\prime}} ; \mathcal{A} \mathbf{w}_{\mathcal{H}}+\nabla r_{\mathcal{H}^{\prime}}\right)_{N, K} \\
F_{\mathcal{H}}^{\prime}\left(\mathbf{v}_{\mathcal{H}}, q_{\mathcal{H}^{\prime}}\right)= & \left(\mathbf{f}, \mathbf{v}_{\mathcal{H}}\right)_{\mathcal{H}}-\sum_{K \in \mathcal{T}_{h}} \tau_{K}\left(\mathcal{B}_{\mathbf{v}_{\mathcal{H}}}+\nabla q_{\mathcal{H}^{\prime}}, \mathbf{f}\right)_{N, K}
\end{aligned}
$$

Then, our analysis allows to prove that the form $B_{\mathcal{H}}^{\prime}$ is stable. This holds because the quadrature formula

$$
\int_{K^{*}} g \mathrm{~d} x^{*} \simeq \sum_{i, j, k=0}^{N} \omega_{i} \omega_{j} \omega_{k} g\left(\xi_{i}, \xi_{j}, \xi_{k}\right)
$$

is exact for $g \in \mathbf{Q}_{2 N-1}\left(K^{*}\right)$.

\section{Solution OF Linear PRIMitive EQUATIONS}

In this section we apply our analysis to the solution of a linear model for the primitive equations of the ocean by a penalty stabilized technique. This model includes the main difficulty of these equations: The vertical convection is degenerated. This makes the pressure to be only in some space $L^{p}$ for $1<p<2$. We prove a discrete inf-sup condition in this norm, and prove the convergence of the approximated solutions to a weak solution of the continuous problem.

To describe our model equations, let us consider a connected $2 \mathrm{D}$ bounded domain $\omega \subset \mathbf{R}^{2}$, and a piecewise continuous function $D: \bar{\omega} \rightarrow \mathbf{R}$ such that $D(\mathbf{x})>0, \forall \mathbf{x}=\left(x_{1}, x_{2}\right) \in \omega$. This function represents the sea depth. We consider the domain

$$
\Omega=\left\{(\mathbf{x}, z) \in \mathbf{R}^{3} \mid \mathbf{x} \in \omega,-D(\mathbf{x})<z<0\right\},
$$

which is intended to represent a piece of the ocean with flat surface. To avoid some technical complexities, we shall assume that $\omega$ is polygonal and $D$ is piecewise affine on some triangulation of $\bar{\omega}$, so that $\Omega$ is polyhedric. Our analysis can be extended to piecewise $C^{1}$ depth functions, similarly to the analysis of the approximation of primitive equations by mixed methods ( $c f$. Chacón Rebollo and Guillén González [12]).

We assume the domain $\Omega$ to be Lipschitz continuous. This occurs, for instance, if the normal derivative of $D$ satisfies $\frac{\partial D}{\partial n} \leq \alpha$ for some $\alpha<0$ a. e. on the part of $\partial \omega$ where $D=0$. Notice that $D$ may be zero partially or totally on $\partial \omega$. Also, that we allow the sea bottom to have vertical walls when $D$ has a jump, and sidewalls if $D>0$ on a part of $\partial \omega$.

We also consider the following subsets of $\partial \Omega$ :

$$
\Gamma_{s}=\left\{(\mathbf{x}, 0) \in \mathbf{R}^{3} \mid \mathbf{x} \in \bar{\omega}\right\}, \quad \text { (sea surface), }
$$

$$
\Gamma_{b}=\partial \Omega-\Gamma_{s} \quad \text { (sea bottom and, eventually, sidewalls) }
$$


We assume known a convection velocity $\mathbf{W}=\left(w_{1}, w_{2}, w_{3}\right)$ on $\Omega$, such that

$$
\left\{\begin{array}{l}
\mathbf{w}=\left(w_{1}, w_{2}\right) \in\left[H^{1}(\Omega)\right]^{2}, w_{3} \in L^{2}(\Omega), \\
\nabla \cdot \mathbf{W}=0 \text { in } \Omega, w_{\left.3\right|_{\Gamma_{s}}}=0, w_{3} \cdot n_{\left.3\right|_{\Gamma_{b}}}=0, \mathbf{w}_{\left.\right|_{\Gamma_{b}}}=0,
\end{array}\right.
$$

where $n_{3}$ denotes the third component of the outward normal to $\partial \Omega, \mathbf{n}=\left(n_{1}, n_{2}, n_{3}\right)$. We are thus forcing the incompressibility of the sea water (Boussinesq's hypothesis). The first boundary condition means that we assume the sea surface to not move in the vertical direction (rigid lid hypothesis), while the second and third ones are rather technical boundary conditions, meaning that we treat the whole $\Gamma_{b}$ as a solid wall.

We also assume known a distributed source term $\mathbf{f}$, representing the effects of temperature, salinity and Coriolis force (assumed to be constant on the whole domain for simplicity), and a "surface wind tension" $\mathrm{g}$. We set the following problem:

$$
\begin{cases}\text { Obtain } \mathbf{y}: \bar{\Omega} \rightarrow \mathbf{R}^{2}, \mathbf{y}=\left(y_{1}, y_{2}\right), & \text { (horizontal velocity) } \\ \text { and } p: \omega \rightarrow \mathbf{R} \text { such that } & \text { (surface pressure) } \\ \mathbf{W} \cdot \nabla \mathbf{y}-\nu \Delta \mathbf{y}+\nabla_{H} p=\mathbf{f} & \text { in } \Omega, \\ \nabla_{H} \cdot\langle\mathbf{y}\rangle=0 & \text { in } \omega, \\ \mathbf{y}_{\left.\right|_{\Gamma_{b}}}=0, \quad \nu \frac{\partial \mathbf{y}}{\partial n_{\Gamma_{s}}}=\mathbf{g} . & \end{cases}
$$

Here, $\nabla_{H}=\left(\partial_{1}, \partial_{2}\right)$ stands for the horizontal gradient, and the symbols $\langle\cdot\rangle$ denote vertical mean,

$$
\langle\mathbf{y}\rangle(\mathbf{x})=\int_{-D(\mathbf{x})}^{0} \mathbf{y}(\mathbf{x}, z) \mathrm{d} z, \quad \text { for } \mathbf{x} \in \omega .
$$

In this problem the surface pressure $p$ acts as a Lagrange multiplier associated to the condition $\nabla_{H} \cdot\langle\mathbf{y}\rangle=0$.

Problem (50) is a reduced version of a linear model of the primitive equations of the ocean (introduced in Lions, Temam and Wang [24]), that reads as follows:

$$
\begin{cases}\text { Obtain a velocity field }\left(\mathbf{y}, y_{3}\right): \bar{\Omega} \rightarrow \mathbf{R}^{3}, & \\ \text { and a pressure } P: \Omega \rightarrow \mathbf{R} \text { such that } & \\ \mathbf{W} \cdot \nabla \mathbf{y}-\nu \Delta \mathbf{y}+\nabla_{H} P=\mathbf{f} & \text { in } \Omega, \\ \nabla \cdot\left(\mathbf{y}, y_{3}\right)=0 & \text { in } \Omega, \\ \partial_{3} P=-\rho g & \text { in } \Omega, \\ \mathbf{y}_{\left.\right|_{\Gamma_{b}}}=0, \quad \nu \frac{\partial \mathbf{y}}{\partial n_{\Gamma_{s}}}=\mathbf{g}, & \\ y_{3} \cdot n_{\left.\right|_{\Gamma_{b}}}=0, \quad y_{\left.\right|_{\Gamma_{s}}}=0 . & \end{cases}
$$

Here, $\rho$ represents the sea water density, assumed to be constant, and $g$ the acceleration of the gravity.

This model is formally obtained from the Navier-Stokes equations by neglecting in the vertical momentum equation all forces (convection, diffusion and Coriolis) but the gravity. This leads to the hydrostatic pressure approximation. A rigorous derivation of this approximation is found in Besson and Laydi [2], as an asymptotic limit as the ratio between vertical and horizontal dimensions tends to zero. The physically meaningful nonlinear - problem would be to find a "fixed point" of equations (50), in the sense that $\mathbf{y}=\mathbf{w}$. This justifies the choice of regularity and boundary conditions satisfied by $\mathbf{w}$ (see (49)).

Equations (50) may be viewed as a model problem for the nonlinear primitive equations, much as the Oseen equations are a linear model for the Navier-Stokes equations. 
Remark 6. Problems (50) and (51) are equivalent. The key point for this equivalence is the following: If a horizontal velocity $\mathbf{y}=\left(y_{1}, y_{2}\right) \in\left[H^{1}(\Omega)\right]^{2}$ satisfies $\mathbf{y}_{\left.\right|_{\Gamma_{b}}}=0$, then

$$
\left\langle\nabla_{H} \cdot \mathbf{y}\right\rangle=\nabla_{H} \cdot\langle\mathbf{y}\rangle
$$

As a consequence, there exists a vertical velocity $y_{3} \in L^{2}(\Omega)$ such that

$$
\nabla \cdot\left(\mathbf{y}, y_{3}\right)=0 \text { in } \Omega, \quad y_{\left.\right|_{\Gamma_{s}}}=0 \text { and } y_{3} \cdot n_{\left.\right|_{\Gamma_{b}}}=0
$$

if and only if

$$
y_{3}\left(\mathbf{x}, x_{3}\right)=\int_{x_{3}}^{0} \nabla_{H} \cdot \mathbf{y}(\mathbf{x}, s) \mathrm{d} s \quad \text { in } \Omega,
$$

and

$$
\nabla_{H} \cdot\langle\mathbf{y}\rangle=0 \text { in } \omega .
$$

This allows to eliminate the vertical velocity $y_{3}$ from problem (51). Also, the condition $\partial_{3} P=-\rho g$ allows to recover the pressure $P$ from the surface pressure $p$, by

$$
P(\mathbf{x}, z)=\rho g z+p(\mathbf{x})
$$

A rigourous proof of this equivalence may be found in Lewandowski [23].

To give a variational formulation to problem (51), let us define the spaces

$$
\begin{gathered}
V_{k}=\left\{\mathbf{v}=\left(v_{1}, v_{2}\right) \in\left[W^{1, k}(\Omega)\right]^{2} \mid \mathbf{v}_{\left.\right|_{\Gamma_{b}}}=0\right\} \quad \text { for } k \geq 1 \text {, integer; } \\
L_{D}^{\alpha}(\omega)=\left\{q: \omega \rightarrow \mathbf{R} \text { measurable such that } \int_{\omega} D(\mathbf{x})|q(\mathbf{x})|^{\alpha} \mathrm{d} x<+\infty\right\} \text { for } \alpha \geq 1 ; \\
L_{D, 0}^{\alpha}(\omega)=L_{D}^{\alpha}(\omega) / \mathbf{R} \text { (quotient space). }
\end{gathered}
$$

Spaces $L_{D}^{\alpha}(\omega)$ and $L_{D, 0}^{\alpha}(\omega)$ are Banach spaces - reflexive if $1<\alpha<+\infty-$, respectively endowed with the norms

$$
\begin{gathered}
\|q\|_{L_{D}^{\alpha}(\omega)}=\left[\int_{\omega} D(\mathbf{x})|q(\mathbf{x})|^{\alpha} \mathrm{d} x\right]^{1 / \alpha}, \\
\|q\|_{L_{D, 0}^{\alpha}(\omega)}=\inf _{c \in \mathbf{R}}\|q+c\|_{L_{D}^{\alpha}(\omega)} .
\end{gathered}
$$

Space $L_{D}^{\alpha}(\omega)$ is isomorphic, and, more specifically, isometric, to the space

$$
L^{\alpha}\left(\partial_{3}, \Omega\right)=\left\{q \in L^{\alpha}(\Omega) \text { such that } \partial_{3} q=0\right\} .
$$

Indeed, we identify each $q \in L_{D}^{\alpha}(\omega)$ with its extension to $\Omega$ as a constant function with respect to the $x_{3}$ variable. Then, we have $\|q\|_{L_{D}^{\alpha}(\omega)}=\|q\|_{L^{\alpha}(\Omega)}$.

Moreover, if we consider the space $L_{0}^{\alpha}\left(\partial_{3}, \Omega\right)=L^{\alpha}\left(\partial_{3}, \Omega\right) / \mathbf{R}$, then $L_{D, 0}^{\alpha}(\omega)$ and $L_{0}^{\alpha}\left(\partial_{3}, \Omega\right)$ also are isomorphic, and $\|q\|_{L_{D, 0}^{\alpha}(\omega)}=\|q\|_{L_{0}^{\alpha}(\Omega)}, \forall q \in L_{D, 0}^{\alpha}(\omega)$.

We further assume $\mathbf{f} \in V_{2}^{\prime}$ and $\mathbf{g} \in\left[H^{-1 / 2}\left(\Gamma_{s}\right)\right]^{d}$, the dual space of $\left[H^{1 / 2}\left(\Gamma_{s}\right)\right]^{d}$. This space is well defined as $\Gamma_{s}$ is $C^{\infty}$. 
We consider the following weak formulation of problem (51):

$$
\left\{\begin{array}{l}
\text { Obtain }(\mathbf{y}, p) \in V_{2} \times L_{D, 0}^{3 / 2}(\omega) \text { such that } \\
B^{(P E)}(\mathbf{y}, p ; \mathbf{v}, q)=F(\mathbf{v}) ; \quad \forall(\mathbf{v}, q) \in V_{4} \times L_{D, 0}^{2}(\omega)
\end{array}\right.
$$

where

$$
\begin{aligned}
& B^{(P E)}(\mathbf{y}, p ; \mathbf{v}, q)=\langle\mathbf{W} \cdot \nabla \mathbf{y}, \mathbf{v}\rangle_{V_{4}^{\prime}-V_{4}}+\nu(\nabla \mathbf{y}, \nabla \mathbf{v})_{\Omega}-\left(p, \nabla_{H} \cdot\langle\mathbf{v}\rangle\right)_{\omega} \\
&-\left(\nabla_{H} \cdot\langle\mathbf{y}\rangle, q\right)_{\omega}, \\
& F(\mathbf{v})=\langle\mathbf{f}, \mathbf{v}\rangle_{V_{2}^{\prime}-V_{2}}+\langle\mathbf{g}, \mathbf{v}\rangle_{H^{-1 / 2}\left(\Gamma_{s}\right)-H^{1 / 2}\left(\Gamma_{s}\right)} .
\end{aligned}
$$

This form is well defined, due to the following:

Lemma 5. The following statements hold.

i) Consider a function $\mathbf{W}=\left(\mathbf{w}, w_{3}\right) \in V_{2} \times L^{2}(\Omega)$ such that $\nabla \cdot \mathbf{W}=0, w_{\left.3\right|_{\Gamma_{s}}}=0$. Then, $\forall \mathbf{u} \in V_{2}$, $\mathbf{W} \cdot \nabla \mathbf{u} \in V_{k}^{\prime}$ for $k \geq 3$, and

$$
\|\mathbf{W} \cdot \nabla \mathbf{u}\|_{V_{k}^{\prime}} \leq \hat{C}_{k}|\mathbf{w}|_{1, \Omega}|\mathbf{u}|_{1, \Omega}
$$

for some constant $\hat{C}_{k}>0$.

ii) If $\mathbf{w} \in V_{k}$ for some $k \geq 1$, then $\langle\mathbf{w}\rangle \in\left[W^{1, k}(\omega)\right]^{2}$ and $\partial_{i}\langle\mathbf{w}\rangle=\left\langle\partial_{i} \mathbf{w}\right\rangle$, i=1,2.

Proof. i) Observe that, given $\mathbf{w} \in V_{2}$, and $w_{3} \in L^{2}(\Omega)$ such that $\partial_{3} w_{3}=-\nabla_{H} \cdot \mathbf{w}$, and $\left.w_{3}\right|_{\Gamma_{s}}=0$ we have $w_{3}\left(\mathbf{x}, x_{3}\right)=\int_{x_{3}}^{0} \nabla_{H} \cdot \mathbf{w}(\mathbf{x}, s) \mathrm{d} s$. Thus,

$$
\left\|w_{3}\right\|_{0, \Omega}+\left\|\partial_{3} w_{3}\right\|_{0, \Omega} \leq C_{1}|\mathbf{w}|_{1, \Omega}
$$

for some constant $C_{1}>0$.

Now, if $\mathbf{W}$ is smooth, we see by integrations by parts that for $\mathbf{u} \in V_{2}$ and $\mathbf{v} \in V_{k}$,

$$
\int_{\Omega}(\mathbf{W} \cdot \nabla \mathbf{u}) \cdot \mathbf{v} \mathrm{d} \mathbf{x} \mathrm{d} x_{3}=\int_{\Omega}\left[\left(\mathbf{w} \cdot \nabla_{H} \mathbf{u}\right) \cdot \mathbf{v}-\partial_{3} w_{3} \mathbf{u} \cdot \mathbf{v}-w_{3} \mathbf{u} \cdot \partial_{3} \mathbf{v}\right] \mathrm{d} \mathbf{x} \mathrm{d} x_{3} .
$$

Then, we may define the duality $\langle\mathbf{W} \cdot \nabla \mathbf{u}, \mathbf{v}\rangle$ by

$$
\langle\mathbf{W} \cdot \nabla \mathbf{u}, \mathbf{v}\rangle=\int_{\Omega}\left[\left(\mathbf{w} \cdot \nabla_{H} \mathbf{u}\right) \cdot \mathbf{v}-\partial_{3} w_{3} \mathbf{u} \cdot \mathbf{v}-w_{3} \mathbf{u} \cdot \partial_{3} \mathbf{v}\right] \mathrm{d} \mathbf{x} \mathrm{d} x_{3}
$$

Using (56),

$$
\begin{aligned}
|\langle\mathbf{W} \cdot \nabla \mathbf{u}, \mathbf{v}\rangle| \leq & C_{2}\left(\|\mathbf{w}\|_{0,4 ; \Omega}|\mathbf{u}|_{1, \Omega}\|\mathbf{v}\|_{0,4 ; \Omega}+|\mathbf{w}|_{1, \Omega}\|\mathbf{u}\|_{0,4 ; \Omega}\|\mathbf{v}\|_{0,4 ; \Omega}\right. \\
& \left.+\|\mathbf{w}\|_{0, \Omega}\|\mathbf{u}\|_{0,6 ; \Omega}\left\|\partial_{3} \mathbf{v}\right\|_{0,3 ; \Omega}\right) \leq \hat{C}_{k}|\mathbf{w}|_{1, \Omega}|\mathbf{u}|_{1, \Omega}|\mathbf{v}|_{1, k ; \Omega} .
\end{aligned}
$$

This proves that $\mathbf{W} \cdot \nabla \mathbf{u} \in V_{k}^{\prime}$. Next, consider a field $\mathbf{W}=\left(\mathbf{w}, w_{3}\right) \in V_{2} \times L^{2}(\Omega)$ with $\nabla \cdot \mathbf{W}=0, w_{\left.3\right|_{\Gamma_{s}}}=0$. Then, there exists a sequence $\left\{\mathbf{w}_{n}\right\}_{n \geq 1} \subset[\mathcal{D}(\bar{\Omega})]^{2}$ such that $\mathbf{w}_{n}=0$ on $\Gamma_{b}$, which converges to $\mathbf{w}$ in $V_{2}$. This is proved by a standard argument (for instance, by symmetrization with respect to $\Gamma_{s}$ ) using that $\partial \Omega$ is Lipschitz-continuous. Let $\mathbf{W}_{n}=\left(\mathbf{w}_{n}, w_{3 n}\right)$, with $w_{3 n}\left(\mathbf{x}, x_{3}\right)=\int_{x_{3}}^{0} \nabla_{H} \cdot \mathbf{w}_{n}(\mathbf{x}, s) \mathrm{d} s$. 
Following Dautray and Lions [18], Chapter XXI, we may ensure that if a function $z \in L^{2}(\Omega)$ is such that $\partial_{3} z \in L^{2}(\Omega)$, then the trace of $z$ on $\Gamma_{s}$ belongs to $H^{1 / 2}\left(\Gamma_{s}\right)$. Moreover, a Poincaré inequality holds if $z_{\left.\right|_{s}}=0$ :

$$
\|z\|_{0, \Omega} \leq C_{3}\left\|\partial_{3} z\right\|_{0, \Omega},
$$

for some constant $C_{3}>0$. Therefore,

$$
\left\|w_{3}-w_{3 n}\right\|_{0, \Omega} \leq C_{3}\left\|\nabla_{H} \cdot\left(\mathbf{w}-\mathbf{w}_{n}\right)\right\|_{0, \Omega},
$$

and $w_{3 n}$ converges to $w_{3}$ in $L^{2}(\Omega)$. Thus, we may pass to the limit in the r.h.s. of (57), and define $\mathbf{W} \cdot \nabla \mathbf{u}$ as a linear form on $V_{k}$. Now, passing to the limit in (58) we deduce $\mathbf{W} \cdot \nabla \mathbf{u} \in V_{k}^{\prime}$ and estimate (55).

ii) Consider $\mathbf{w} \in V_{k}$. As $\mathbf{w} \in\left[L^{k}(\Omega)\right]^{2}$, one readily proves $\langle\mathbf{w}\rangle \in\left[L^{k}(\omega)\right]^{2}$. Also, let $\varphi \in \mathcal{D}(\omega)$. Then, if $\mathbf{w}$ is smooth, for $i=1,2$,

$$
\begin{aligned}
\int_{\omega}\left\langle\partial_{i} \mathbf{w}\right\rangle(\mathbf{x}) \varphi(\mathbf{x}) \mathrm{d} \mathbf{x} & =\int_{\Omega} \partial_{i} \mathbf{w}\left(\mathbf{x}, x_{3}\right) \varphi(\mathbf{x}) \mathrm{d} \mathbf{x} \mathrm{d} x_{3}= \\
& =\int_{\partial \Omega} n_{i} \mathbf{w} \varphi \mathrm{d}(\partial \Omega)-\int_{\Omega} \mathbf{w}\left(\mathbf{x}, x_{3}\right) \partial_{i} \varphi(\mathbf{x}) \mathrm{d} \mathbf{x} \mathrm{d} x_{3}= \\
& =-\int_{\omega}\langle\mathbf{w}\rangle(\mathbf{x}) \partial_{i} \varphi(\mathbf{x}) \mathrm{d} \mathbf{x},=-\int_{\omega} \partial_{i}\langle\mathbf{w}\rangle(\mathbf{x}) \varphi(\mathbf{x}) \mathrm{d} \mathbf{x},
\end{aligned}
$$

as $n_{i}=0$ on $\Gamma_{s}$ and $\mathbf{w}=0$ on $\Gamma_{b}$. Thus, $\partial_{i}\langle\mathbf{w}\rangle=\left\langle\partial_{i} \mathbf{w}\right\rangle$ and $\mathbf{w} \in\left[W^{1, k}(\omega)\right]^{2}$.

If $\mathbf{w}$ is any element of $V_{k}$, the same results follows from a density argument similar to that of the proof of statement i) above.

Remark 7. Any solution ( $\mathbf{y}, p)$ of problem (54) is a weak solution of problem (50) in the distribution sense. Furthermore, if we recover the vertical velocity $y_{3}$ by (52), and the physical pressure $P$ by (53), then the couple $\left(\left(\mathbf{y}, y_{3}\right), P\right)$ is a solution of problem (51) in the distribution sense.

We shall discretize problem (54) by a penalty stabilized method, of Brezzi and Pitkäranta's kind (cf. [9]). Consider a triangulation $\mathcal{C}_{h}$ of $\bar{\omega}$ such that $D$ is affine on each triangle $T \in \mathcal{C}_{h}$. Consider also a partition $\mathcal{P}_{h}$ of $\bar{\Omega}$ by sets of the form

$$
P_{T}=\left\{\left(\mathbf{x}, x_{3}\right) \in \mathbf{R}^{3}, \text { such that } \mathbf{x} \in T,-D(\mathbf{x}) \leq x_{3} \leq 0\right\} \text { for some triangle } T \in \mathcal{C}_{h} .
$$

Notice that if a triangle $T \in \mathcal{C}_{h}$ is not adjacent to $\partial \omega$, or if it is adjacent to $\partial \omega$ and $D>0$ on $\bar{T}$, then its associated set $P_{T}$ is a triangular prism with upper base $T \times\{0\}$ and possibly non-horizontal lower base. However, if $T$ is adjacent to $\partial \omega$ and $D=0$ on a part of $\partial T$, then $P_{T}$ is a non-prismatic polyhedron.

We shall consider a triangulation $\mathcal{T}_{h}$ of $\Omega$ constructed by subdividing each element $\mathcal{P}_{h}$ into tetrahedra. Let us define the finite element spaces,

$$
\begin{gathered}
V_{h}=\left\{v_{h} \in C^{0}(\bar{\Omega}) \mid v_{\left.h\right|_{K}} \in \mathbf{P}_{1}(K), \forall K \in \mathcal{T}_{h}\right\} ; \\
Y_{h}=\left\{\mathbf{v}_{h} \in V_{h}^{2} \mid v_{\left.h\right|_{\Gamma_{b}}}=0\right\} ; \\
\tilde{N}_{h}=\left\{q_{h} \in C^{0}(\bar{\omega}) \mid q_{\left.\right|_{\mid}} \in \mathbf{P}_{1}(T), \forall T \in \mathcal{C}_{h}\right\} ; \quad N_{h}=\tilde{N}_{h} / R .
\end{gathered}
$$

We introduce the following discretization of (54):

$$
\left\{\begin{array}{l}
\text { Obtain }\left(\mathbf{y}_{h}, p_{h}\right) \in Y_{h} \times N_{h} \text { such that } \\
B_{h}^{(P E)}\left(\mathbf{y}_{h}, p_{h} ; \mathbf{v}_{h}, q_{h}\right)=F\left(\mathbf{v}_{h}\right) ; \quad \forall\left(\mathbf{v}_{h}, q_{h}\right) \in Y_{h} \times N_{h}
\end{array}\right.
$$


where

$$
\begin{aligned}
B_{h}^{(P E)}\left(\mathbf{u}_{h}, r_{h} ; \mathbf{v}_{h}, q_{h}\right)= & B^{(P E)}\left(\mathbf{u}_{h}, r_{h} ; \mathbf{v}_{h}, q_{h}\right)+\sum_{K \in \mathcal{T}_{h}} \tau_{K}^{(c)}\left(\mathbf{W}_{h} \cdot \nabla \mathbf{u}_{h}, \mathbf{W}_{h} \cdot \nabla \mathbf{v}_{h}\right)_{K} \\
& -\sum_{T \in \mathcal{C}_{h}} \tau_{T}^{(p)}\left(\nabla_{H} r_{h}, \nabla_{H} q_{h}\right)_{T} .
\end{aligned}
$$

The stabilizing coefficients for convection $\tau_{K}^{(c)}$ are assumed to be still given by (11). This will provide some stabilization of the convective derivative. Also, to ensure the stability of the pressure discretization we shall assume that the stabilizing coefficients for pressure $\tau_{T}^{(p)}$ satisfy the following condition: There exist two constants $\beta_{1}>0, \beta_{2}>0$ such that

$$
\beta_{1} h_{T}^{2} \frac{\int_{T} D \mathrm{~d} \mathbf{x}}{|T|} \leq \tau_{T}^{(p)} \leq \beta_{2} h_{T}^{2} \frac{\int_{T} D \mathrm{~d} \mathbf{x}}{|T|}, \quad \forall T \in \mathcal{C}_{h}
$$

Observe that these inequalities make sense as we assume $D>0$ on $\omega$. In the stabilizing terms of (63), we replace the convection velocity $\mathbf{W}=\left(\mathbf{w}, w_{3}\right)$ by some interpolate $\mathbf{W}_{h}=\left(\mathbf{w}_{h}, w_{3 h}\right) \in Y_{h} \times V_{h}$, satisfying for some constant $C>0$,

$$
\left|\mathbf{W}_{h}\right|_{1} \leq C|\mathbf{w}|_{1}
$$

We now state the main result of this section.

Theorem 6. Assume the convection velocity $\mathbf{W}=\left(\mathbf{w}, w_{3}\right)$ lies in the space $V_{2} \times L^{2}(\Omega)$ and verifies $\nabla \cdot \mathbf{W}=0$, $w_{\left.3\right|_{\Gamma_{s}}}=0$. Assume the triangulations $\left\{\mathcal{T}_{h}\right\}_{h>0}$ are regular. Then, the following statements hold.

i) Problem (63) admits a unique solution $\left(\mathbf{y}_{h}, p_{h}\right) \in Y_{h} \times N_{h}$ which is bounded in $V_{2} \times L_{D, 0}^{3 / 2}(\omega)$

ii) The sequence $\left\{\left(\mathbf{y}_{h}, p_{h}\right)\right\}_{h>0}$ contains a subsequence which is weakly convergent in $V_{2} \times L_{D, 0}^{3 / 2}(\omega)$ to a solution of (54) satisfying the estimate

$$
|\mathbf{y}|_{1}+\|p\|_{L_{D, 0}^{3 / 2}(\omega)} \leq C\left(\|\mathbf{f}\|_{V_{2}^{\prime}}+\|\mathbf{g}\|_{-1 / 2, \Gamma_{s}}\right)\left(1+|\mathbf{w}|_{1, \Omega}\right)
$$

for some constant $C>0$ independent of $h$.

Proof. We proceed by steps.

\section{Step 1: Embedding of method (63) in abstract method.}

Given an element $T \in \mathcal{C}_{h}$, let us define $\tau_{K}^{(p)}=\frac{|T|}{\int_{T} D \mathrm{~d} \mathbf{x}} \tau_{T}^{(p)}$, for any element $K \in \mathcal{T}_{h}$ that be in the prism $P_{T}$ that lies on $T$. We assume that the pressures of $N_{h}^{T}$ are defined on the whole $\Omega$, as constant functions in the $x_{3}$ 
variable. Then,

$$
\begin{aligned}
\sum_{K \in \mathcal{T}_{h}} \tau_{K}^{(p)}\left(\nabla r_{h}, \nabla q_{h}\right)_{K} & =\sum_{T \in \mathcal{C}_{h}} \frac{|T|}{\int_{T} D \mathrm{~d} \mathbf{x}} \tau_{T}^{(p)} \int_{P_{T}} \nabla_{H} r_{h} \cdot \nabla_{H} q_{h} \mathrm{~d} \mathbf{x} \mathrm{d} x_{3} \\
& =\sum_{T \in \mathcal{C}_{h}} \frac{|T|}{\int_{T} D \mathrm{~d} \mathbf{x}} \tau_{T}^{(p)}\left(\nabla_{H} r_{h}\right)_{\left.\right|_{T}} \cdot\left(\nabla_{H} q_{h}\right)_{\left.\right|_{T}} \int_{P_{T}} \mathrm{~d} \mathbf{x} \mathrm{d} x_{3} \\
& =\sum_{T \in \mathcal{C}_{h}} \tau_{T}^{(p)}\left(\nabla_{H} r_{h}, \nabla_{H} q_{h}\right)_{T}, \quad \forall r_{h}, q_{h} \in N_{h} .
\end{aligned}
$$

Let us define $M_{h}=V_{h} / \mathbf{R}$, where $V_{h}$ is given by (62). We now apply Lemma 1: There exists a bubble finite element space $B_{1 h}$, generated on $\mathcal{T}_{h}$ by a reference element $B_{1}^{*} \subset\left[H_{0}^{1}\left(K^{*}\right)\right]^{3}$, and a bilinear coercive form $\mathbf{S}_{1 h}$ on $\left[H_{0}^{1}(\Omega)\right]^{3}$, such that

$$
\sum_{K \in \mathcal{T}_{h}} \tau_{K}^{(p)}\left(\nabla r_{h}, \nabla q_{h}\right)_{K}=\mathbf{S}_{1 h}\left(\mathcal{R}_{1 h}\left(\nabla r_{h}\right), \mathcal{R}_{1 h}\left(\nabla q_{h}\right)\right), \quad \forall r_{h}, q_{h} \in M_{h}
$$

where $\mathcal{R}_{1 h}$ is the static condensation operator on $B_{1 h}$ with respect to form $\mathbf{S}_{1 h}$. We may identify $N_{h}$ with the subspace of $M_{h}$ defined by $\left\{q_{h} \in V_{h} \mid \partial_{3} q_{h}=0\right\}$. Then, from (67) and (68) we deduce

$$
\sum_{T \in \mathcal{C}_{h}} \tau_{T}^{(p)}\left(\nabla_{H} r_{h}, \nabla_{H} q_{h}\right)_{T}=\mathbf{S}_{1 h}\left(\mathcal{R}_{1 h}\left(\nabla r_{h}\right), \mathcal{R}_{1 h}\left(\nabla q_{h}\right)\right), \quad \forall r_{h}, q_{h} \in N_{h}
$$

Also, again by Lemma 1, there exists a bubble finite element space $B_{2 h}$, generated on $\mathcal{T}_{h}$ by a reference element $B_{2}^{*} \subset\left[H_{0}^{1}\left(K^{*}\right)\right]^{2}$, and a bilinear coercive form $\mathbf{S}_{2 h}$ on $\left[H_{0}^{1}(\Omega)\right]^{2}$, such that $\forall \mathbf{u}_{h}, \mathbf{v}_{h} \in Y_{h}$,

$$
\sum_{K \in \mathcal{T}_{h}} \tau_{K}^{(c)}\left(\mathbf{W}_{h} \cdot \nabla \mathbf{u}_{h}, \mathbf{W}_{h} \cdot \nabla \mathbf{v}_{h}\right)_{K}=\mathbf{S}_{2 h}\left(\mathcal{R}_{2 h}\left(\mathbf{W}_{h} \cdot \nabla \mathbf{u}_{h}\right), \mathcal{R}_{2 h}\left(\mathbf{W}_{h} \cdot \nabla \mathbf{v}_{h}\right)\right)
$$

where $\mathcal{R}_{2 h}$ is the static condensation operator on $B_{2 h}$ with respect to form $\mathbf{S}_{2 h}$. Then,

$$
\begin{aligned}
B_{h}^{(P E)}\left(\mathbf{u}_{h}, r_{h} ; \mathbf{v}_{h}, q_{h}\right)= & B^{(P E)}\left(\mathbf{u}_{h}, r_{h} ; \mathbf{v}_{h}, q_{h}\right) \\
& +\mathbf{S}_{2 h}\left(\mathcal{R}_{2 h}\left(\mathbf{W}_{h} \cdot \nabla \mathbf{u}_{h}\right), \mathcal{R}_{2 h}\left(\mathbf{W}_{h} \cdot \nabla \mathbf{v}_{h}\right)\right) \\
& -\mathbf{S}_{1 h}\left(\mathcal{R}_{1 h}\left(\nabla r_{h}\right), \mathcal{R}_{1 h}\left(\nabla q_{h}\right)\right), \quad \forall \mathbf{u}_{h}, \mathbf{v}_{h} \in Y_{h}, \forall r_{h}, q_{h} \in N_{h} .
\end{aligned}
$$

We recall that by Theorem (2) (Step 3), the forms $\left\{\mathbf{S}_{2 h}\right\}_{h>0}$ are uniformly continuous and coercive in $H^{1}$ norm. Also, due to (64) and the regularity of triangulations $\mathcal{T}_{h}$, the coefficients $\tau_{K}^{(p)}$ are of order $h_{K}^{2}$. Then, the forms $\left\{\mathbf{S}_{1 h}\right\}_{h>0}$ also are uniformly continuous and coercive.

\section{Step 2: Discrete inf-sup condition.}

We state the following:

Lemma 6. Given $\alpha \in(1,2]$, there exists a constant $C_{\alpha}>0$ such that $\forall q_{h} \in N_{h}$,

$$
C_{\alpha}\left\|q_{h}\right\|_{L_{D, 0}^{\alpha}(\omega)} \leq \sup _{\mathbf{v}_{h} \in Y_{h}-\{0\}} \frac{\left(\nabla_{H} \cdot\left\langle\mathbf{v}_{h}\right\rangle, q_{h}\right)_{\omega}}{\left|\mathbf{v}_{h}\right|_{1, \alpha^{\prime}, \Omega}}+\left[\mathbf{S}_{1 h}\left(\mathcal{R}_{1 h}\left(\nabla q_{h}\right), \mathcal{R}_{1 h}\left(\nabla q_{h}\right)\right)\right]^{1 / 2}
$$

where $\alpha^{\prime}$ is the conjugate exponent of $\alpha$. 
Proof. Define the space $W_{h}=\left\{\left(\mathbf{v}_{h}, v_{3 h}\right) \in V_{h}^{3} \mid\left(\mathbf{v}_{h}, v_{3 h}\right)_{\left.\right|_{\partial \Omega}}=0\right\}$. It is enough to prove that

$$
\begin{aligned}
C_{\alpha}\left\|q_{h}\right\|_{L_{0}^{\alpha}(\Omega)} \leq & \sup _{\left(\mathbf{v}_{h}, v_{3 h}\right) \in W_{h}-\{0\}} \frac{\left(\nabla \cdot\left(\mathbf{v}_{h}, v_{3 h}\right), q_{h}\right)_{\Omega}}{\left|\left(\mathbf{v}_{h}, v_{3 h}\right)\right|_{1, \alpha^{\prime}, \Omega}} \\
& +\left[\mathbf{S}_{1 h}\left(\mathcal{R}_{1 h}\left(\nabla q_{h}\right), \mathcal{R}_{1 h}\left(\nabla q_{h}\right)\right)\right]^{1 / 2}, \forall q_{h} \in V_{h} .
\end{aligned}
$$

Indeed, if $q_{h} \in N_{h},\left(\mathbf{v}_{h}, v_{3 h}\right) \in W_{h}$,

$$
\left(\nabla \cdot\left(\mathbf{v}_{h}, v_{3 h}\right), q_{h}\right)_{\Omega}=\left(\nabla_{H} \cdot \mathbf{v}_{h}, q_{h}\right)_{\Omega}-\left(v_{3 h}, \partial_{3} q_{h}\right)_{\Omega}=\left(\nabla_{H} \cdot\left\langle\mathbf{v}_{h}\right\rangle, q_{h}\right)_{\omega} .
$$

Then,

$$
\begin{aligned}
\sup _{\left(\mathbf{v}_{h}, v_{3 h}\right) \in W_{h}-\{0\} \frac{\left(\nabla \cdot\left(\mathbf{v}_{h}, v_{3 h}\right), q_{h}\right)_{\Omega}}{\left|\left(\mathbf{v}_{h}, v_{3 h}\right)\right|_{1, \alpha^{\prime}, \Omega}}} & =\sup _{\left(\mathbf{v}_{h}, v_{3 h}\right) \in W_{h}-\{0\}} \frac{\left(\nabla_{H} \cdot\left\langle\mathbf{v}_{h}\right\rangle, q_{h}\right)_{\omega}}{\left|\left(\mathbf{v}_{h}, v_{3 h}\right)\right|_{1, \alpha^{\prime}, \Omega}} \\
& \leq \sup _{\mathbf{v}_{h} \in Y_{h}-\{0\}} \frac{\left(\nabla_{H} \cdot\left\langle\mathbf{v}_{h}\right\rangle, q_{h}\right)_{\omega}}{\left|\mathbf{v}_{h}\right|_{1, \alpha^{\prime}, \Omega}} .
\end{aligned}
$$

Also, $\left\|q_{h}\right\|_{L_{0}^{\alpha}(\Omega)}=\left\|q_{h}\right\|_{L_{D, 0}^{\alpha}(\omega)}$ if $q_{h} \in N_{h}$. Thus, (71) follows from (72).

To prove (72), consider $q_{h} \in V_{h}$. As $\Omega$ is polyhedric, then $\partial \Omega$ is Lipschitz, and the continuous inf-sup condition in $L^{\alpha}(\Omega)$ norm is satisfied ( $c f$. Amrouche and Girault [1]): There exists a constant $D_{\alpha}>0$ such that

$$
D_{\alpha}\left\|q_{h}\right\|_{L_{0}^{\alpha}(\Omega)} \leq \sup _{\mathbf{v} \in\left[W_{0}^{1, \alpha^{\prime}}(\Omega)\right]^{3}-\{0\}} \frac{\left(\nabla \cdot \mathbf{v}, q_{h}\right)_{\Omega}}{|\mathbf{v}|_{1, \alpha^{\prime}, \Omega}}, \quad \forall q \in L_{0}^{\alpha}(\Omega) .
$$

As $[\mathcal{D}(\Omega)]^{3}$ is dense in $\left[W_{0}^{1, \alpha^{\prime}}(\Omega)\right]^{3}$, there exists a $\mathbf{v}_{0} \in[\mathcal{D}(\Omega)]^{3}$ such that

$$
\frac{1}{2} D_{\alpha}\left\|q_{h}\right\|_{L_{0}^{\alpha}(\Omega)} \leq\left(\nabla \cdot \mathbf{v}_{0}, q_{h}\right)_{\Omega}, \quad\left|\mathbf{v}_{0}\right|_{1, \alpha^{\prime}, \Omega}=1
$$

Following the standard finite elements interpolation theory ( $c f$. Ciarlet [13]), there exists an interpolate $\mathbf{v}_{0 h} \in$ $W_{h}$ such that

$$
\begin{aligned}
\left|\mathbf{v}_{0 h}\right|_{1, \alpha^{\prime}, \Omega} & \leq C_{1}\left|\mathbf{v}_{0}\right|_{1, \alpha^{\prime}, \Omega} \\
\left\|\mathbf{v}_{0 h}-\mathbf{v}_{0}\right\|_{0, K} & \leq C_{1} h_{K}\left|\mathbf{v}_{0}\right|_{1, K}, \quad \forall K \in \mathcal{T}_{h}
\end{aligned}
$$

for some constant $C_{1}>0$ independent of $h$. Then, as $q_{h}$ is continuous,

$$
\begin{aligned}
\frac{1}{2} D_{\alpha}\left\|q_{h}\right\|_{L_{0}^{\alpha}(\Omega)} \leq & \left(\nabla \cdot \mathbf{v}_{0 h}, q_{h}\right)_{\Omega}+\left(\mathbf{v}_{0 h}-\mathbf{v}_{0}, \nabla q_{h}\right)_{\Omega} \\
\leq & C_{1} \frac{\left|\left(\nabla \cdot \mathbf{v}_{0 h}, q_{h}\right)_{\Omega}\right|}{\left|\mathbf{v}_{0 h}\right|_{1, \alpha^{\prime}, \Omega}} \\
& +\left[\sum_{K \in \mathcal{T}_{h}} h_{K}^{-2}\left\|\mathbf{v}_{0 h}-\mathbf{v}_{0}\right\|_{0, K}^{2}\right]^{1 / 2}\left[\sum_{K \in \mathcal{T}_{h}} h_{K}^{2}\left\|\nabla q_{h}\right\|_{0, K}^{2}\right]^{1 / 2}
\end{aligned}
$$

As $\alpha^{\prime} \geq 2$, then (74) yields

$$
\left[\sum_{K \in \mathcal{T}_{h}} h_{K}^{-2}\left\|\mathbf{v}_{0 h}-\mathbf{v}_{0}\right\|_{0, K}^{2}\right]^{1 / 2} \leq C_{2}\left|\mathbf{v}_{0}\right|_{1, \alpha^{\prime}, \Omega}
$$


Also, from the representation formula (68), hypothesis (64) and the regularity of the triangulations, we obtain

$$
\sum_{K \in \mathcal{T}_{h}} h_{K}^{2}\left\|\nabla q_{h}\right\|_{0, K}^{2} \leq C_{3} \mathbf{S}_{1 h}\left(\mathcal{R}_{1 h}\left(\nabla q_{h}\right), \mathcal{R}_{1 h}\left(\nabla q_{h}\right)\right) .
$$

Thus, estimate (72) follows.

\section{Step 3: Existence of solution of discrete problem.}

Problem (63) is equivalent to a square linear system of dimension $\operatorname{dim}\left(Y_{h}\right)+\operatorname{dim}\left(N_{h}\right)$. Then, the existence of solution follows from its uniqueness. If we prove that any solution is bounded by a norm of the data, the uniqueness follows. Let us then consider a solution $\left(\mathbf{y}_{h}, p_{h}\right) \in Y_{h} \times N_{h}$ of problem (63).

Velocity estimate. Denote $\mathbf{c}_{h}=\mathcal{R}_{1 h}\left(\nabla p_{h}\right) \in B_{1 h}, \mathbf{d}_{h}=\mathcal{R}_{2 h}\left(\mathbf{W}_{h} \cdot \nabla \mathbf{y}_{h}\right) \in B_{2 h}$. As $\nabla \cdot \mathbf{W}=0, w_{\left.3\right|_{\Gamma_{s}}}=0$, $\mathbf{y}_{h} \in\left[W^{1, \infty}(\Omega)\right]^{2}$, using (57) we have

$$
\langle\mathbf{W} \cdot \nabla \mathbf{y}, \mathbf{y}\rangle_{V_{4}^{\prime}-V_{4}}=\frac{1}{2} \int_{\partial \Omega} \mathbf{W} \cdot n|\mathbf{y}|^{2} \mathrm{~d}(\partial \Omega)-\frac{1}{2} \int_{\Omega} \nabla \cdot \mathbf{W}|\mathbf{y}|^{2} \mathrm{~d} \mathbf{x} \mathrm{d} x_{3}=0 .
$$

Then,

$$
\nu\left|\mathbf{y}_{h}\right|_{1, \Omega}^{2}+\mathbf{S}_{1 h}\left(\mathbf{c}_{h}, \mathbf{c}_{h}\right)+\mathbf{S}_{2 h}\left(\mathbf{d}_{h}, \mathbf{d}_{h}\right)=B_{h}^{(P E)}\left(\mathbf{y}_{h}, p_{h} ; \mathbf{y}_{h},-p_{h}\right)=F\left(\mathbf{y}_{h}\right) \leq\|F\|_{V_{2}^{\prime}}\left|\mathbf{y}_{h}\right|_{1, \Omega},
$$

where we assume $V_{2}$ to be endowed with the $|\cdot|_{1, \Omega}$ norm. Then,

$$
\left|\mathbf{y}_{h}\right|_{1, \Omega} \leq \nu^{-1}\|F\|_{V_{2}^{\prime}}, \quad \mathbf{S}_{1 h}\left(\mathbf{c}_{h}, \mathbf{c}_{h}\right) \leq \nu^{-1}\|F\|_{V_{2}^{\prime}}^{2}, \quad\left|\mathbf{d}_{h}\right|_{1, \Omega} \leq\left(\nu \nu_{2}\right)^{-1 / 2}\|F\|_{V_{2}^{\prime}},
$$

where $\nu_{2}$ is the uniform coerciveness constant of the forms $\mathbf{S}_{2 h}$.

Pressure estimate. Take $\mathbf{v}_{h} \in Y_{h}$. Then,

$$
\begin{aligned}
\left(\nabla_{H} \cdot\left\langle\mathbf{v}_{h}\right\rangle, p_{h}\right)_{\omega}= & -F\left(\mathbf{v}_{h}\right)+\left\langle\mathbf{W} \cdot \nabla \mathbf{y}_{h}, \mathbf{v}_{h}\right\rangle_{V_{4}^{\prime}-V_{4}}+\nu\left(\nabla \mathbf{y}_{h}, \nabla \mathbf{v}_{h}\right)_{\Omega} \\
& +\mathbf{S}_{2 h}\left(\mathbf{d}_{h}, \mathcal{R}_{2 h}\left(\mathbf{W}_{h} \cdot \nabla \mathbf{v}_{h}\right)\right) .
\end{aligned}
$$

Observe that, due to Sobolev's injections and the stability interpolate (65),

$$
\begin{aligned}
\mathbf{S}_{2 h}\left(\mathbf{d}_{h}, \mathcal{R}_{2 h}\left(\mathbf{W}_{h} \cdot \nabla \mathbf{v}_{h}\right)\right)= & \left(\mathbf{W}_{h} \cdot \nabla \mathbf{v}_{h}, \mathbf{d}_{h}\right)_{\Omega} \leq C_{1}\left\|\mathbf{w}_{h}\right\|_{0,4 ; \Omega}\left|\mathbf{v}_{h}\right|_{1, \Omega}\left\|\mathbf{d}_{h}\right\|_{0,4 ; \Omega} \\
& +C_{2}\left\|w_{3}\right\|_{0, \Omega}\left\|\partial_{3} \mathbf{v}_{h}\right\|_{0,3 ; \Omega}\left\|\mathbf{d}_{h}\right\|_{0,6 ; \Omega} \\
\leq & C_{3}|\mathbf{w}|_{1, \Omega}\left|\mathbf{v}_{h}\right|_{1,3 ; \Omega}\left\|\mathbf{d}_{h}\right\|_{0,6 ; \Omega}
\end{aligned}
$$

Then, due to (55),

$$
\begin{aligned}
\left(\nabla_{H} \cdot\left\langle\mathbf{v}_{h}\right\rangle, p_{h}\right)_{\omega} \leq & C_{4}\left(\|F\|_{V_{2}^{\prime}}+|\mathbf{w}|_{1, \Omega}\left|\mathbf{y}_{h}\right|_{1, \Omega}+\nu\left|\mathbf{y}_{h}\right|_{1, \Omega}\right. \\
& \left.+|\mathbf{w}|_{1, \Omega}\left|\mathbf{d}_{h}\right|_{1, \Omega}\right)\left|\mathbf{v}_{h}\right|_{1,3 ; \Omega} .
\end{aligned}
$$

Then, using (75),

$$
\sup _{\mathbf{v}_{h} \in Y_{h}-\{0\}} \frac{\left(\nabla_{H} \cdot\left\langle\mathbf{v}_{h}\right\rangle, q_{h}\right)_{\omega}}{\left|\mathbf{v}_{h}\right|_{1,3, \Omega}}+\left[\mathbf{S}_{1 h}\left(\mathbf{c}_{h}, \mathbf{c}_{h}\right)\right]^{1 / 2} \leq C_{5}\|F\|_{V_{2}^{\prime}}\left(1+|\mathbf{w}|_{1, \Omega}\right) .
$$

Due to Lemma 6, we deduce that

$$
\left\|p_{h}\right\|_{L_{D, 0}^{3 / 2}(\omega)} \leq C_{6}\|F\|_{V_{2}^{\prime}}\left(1+|\mathbf{w}|_{1, \Omega}\right) .
$$


Thus, the discrete problem (54) admits a unique solution satisfying

$$
\left|\mathbf{y}_{h}\right|_{1}+\left\|p_{h}\right\|_{L_{D, 0}^{3 / 2}(\omega)} \leq C_{7}\|F\|_{V_{2}^{\prime}}\left(1+|\mathbf{w}|_{1, \Omega}\right)
$$

\section{Step 4: Conclusion.}

Due to estimates (77), the sequence $\left\{\left(\mathbf{y}_{h}, p_{h}\right)\right\}_{h>0}$ is bounded in $V_{2} \times L_{D, 0}^{3 / 2}(\omega)$, which is a reflexive space. Then, it contains a subsequence, that we still denote in the same way, weakly convergent in that space to a pair $(\mathbf{y}, p)$. Let us prove that this pair is a solution of problem (50).

Consider a pair $(\mathbf{v}, q) \in V_{4} \times L_{D, 0}^{2}(\omega)$. There exists a sequence $\left\{\left(\mathbf{v}_{h}, q_{h}\right)\right\}_{h>0}$ with $\left(\mathbf{v}_{h}, q_{h}\right) \in Y_{h} \times N_{h}$ which is strongly convergent to $(\mathbf{v}, q)$ in $V_{4} \times L_{D, 0}^{2}(\omega)$. Indeed, the fact that $\mathbf{v}_{h} \rightarrow \mathbf{v}$ in $V_{4}$ is proved by the standard interpolation estimates by piecewise affine finite elements. Also, by the same theory, there exists a sequence $\left\{q_{h}\right\}_{h>0}$ with $q_{h} \in \tilde{N}_{h}$ which converges to $q$ in $L^{2}(\omega)$. But

$$
\left\|q_{h}-q\right\|_{L_{D, 0}^{2}(\omega)} \leq\left\|q_{h}-q\right\|_{L_{D}^{2}(\omega)} \leq\|D\|_{0, \infty ; \omega}^{1 / 2}\left\|q_{h}-q\right\|_{0,2 ; \omega} .
$$

Thus, $\lim _{h \rightarrow 0}\left\|q_{h}-q\right\|_{L_{D, 0}^{2}(\omega)}=0$.

From (57) we deduce

$$
\lim _{h \rightarrow 0}\left\langle\mathbf{W} \cdot \nabla \mathbf{y}_{h}, \mathbf{v}_{h}\right\rangle_{V_{4}^{\prime}-V_{4}}=\langle\mathbf{W} \cdot \nabla \mathbf{y}, \mathbf{v}\rangle_{V_{4}^{\prime}-V_{4}}
$$

Also,

$$
\lim _{h \rightarrow 0}\left(p_{h}, \nabla_{H} \cdot\left\langle\mathbf{v}_{h}\right\rangle\right)_{\omega}=\lim _{h \rightarrow 0}\left(p_{h}, \nabla_{H} \cdot \mathbf{v}_{h}\right)_{\Omega}=\left(p, \nabla_{H} \cdot \mathbf{v}\right)_{\Omega}=\left(p, \nabla_{H} \cdot\langle\mathbf{v}\rangle\right)_{\omega}
$$

and similarly

$$
\lim _{h \rightarrow 0}\left(\nabla_{H} \cdot\left\langle\mathbf{y}_{h}\right\rangle, q_{h}\right)_{\omega}=\left(\nabla_{H} \cdot\langle\mathbf{y}\rangle, q\right)_{\omega}
$$

In a standard way, we have

$$
\lim _{h \rightarrow 0}\left(\nabla \mathbf{y}_{h}, \nabla \mathbf{v}_{h}\right)_{\Omega}=(\nabla \mathbf{y}, \nabla \mathbf{v})_{\Omega}
$$

To pass to the limit in the stabilizing terms, we need the following property of the bubble finite element spaces:

Lemma 7. Consider a family $\left\{Z_{h}\right\}_{h>0}$ of finite element subspaces of $\left[H_{0}^{1}(\Omega)\right]^{d}$ generated by a reference space $Z^{*}$. Assume that $Z^{*}$ does not contain the constant functions. Then, the following statements hold.

i) For any $q \in[2,6]$, there exists a constant $C_{q}>0$ such that

$$
\left\|\mathbf{z}_{h}\right\|_{0, q} \leq C_{q} h^{\beta}\left|\mathbf{z}_{h}\right|_{1}, \quad \forall \mathbf{z}_{h} \in Z_{h} ; \quad \text { where } \frac{\beta}{2}+\frac{1-\beta}{6}=\frac{1}{q} .
$$

ii) If a sequence $\left\{\mathbf{z}_{h}\right\}_{h>0}$, with $\mathbf{z}_{h} \in Z_{h}, \quad \forall h>0$, is bounded in $\left[H_{0}^{1}(\Omega)\right]^{d}$, then it converges weakly to zero in $\left[H_{0}^{1}(\Omega)\right]^{d}$.

This Lemma is proved in the Appendix.

Due to (75) and to the uniform coerciveness of the forms $\mathbf{S}_{1 h}$, the sequence $\left\{\mathbf{c}_{h}\right\}_{h>0}$ is bounded in $\left[H_{0}^{1}(\Omega)\right]^{3}$. Then, it is weakly convergent to zero in that space. Thus,

$$
\lim _{h \rightarrow 0} \mathbf{S}_{1 h}\left(\mathcal{R}_{1 h}\left(\nabla q_{h}\right), \mathbf{c}_{h}\right)=\lim _{h \rightarrow 0}\left(-q_{h}, \nabla \cdot \mathbf{c}_{h}\right)_{\Omega}=0 .
$$


Also

$$
\begin{aligned}
\left|\mathbf{S}_{2 h}\left(\mathbf{d}_{h}, \mathcal{R}_{2 h}\left(\mathbf{W}_{h} \cdot \nabla \mathbf{v}_{h}\right)\right)\right| & =\left|\left\langle\mathbf{W}_{h} \cdot \nabla \mathbf{v}_{h}, \mathbf{d}_{h}\right\rangle_{H^{-1}-H_{0}^{1}}\right|=\left|\int_{\Omega}\left(\mathbf{W}_{h} \cdot \nabla \mathbf{v}_{h}\right) \cdot \mathbf{d}_{h} \mathrm{~d} \mathbf{x} \mathrm{d} x_{3}\right| \\
& \leq C_{7}\left(\left\|\mathbf{w}_{h}\right\|_{0,4 ; \Omega}\left|\mathbf{v}_{h}\right|_{1, \Omega}\left\|\mathbf{d}_{h}\right\|_{0,4 ; \Omega}+\left\|w_{3 h}\right\|_{0, \Omega}\left\|\partial_{3} \mathbf{v}_{h}\right\|_{0,4 ; \Omega}\left\|\mathbf{d}_{h}\right\|_{0,4 ; \Omega}\right) \\
& \leq C_{8}|\mathbf{w}|_{1, \Omega}\left|\mathbf{v}_{h}\right|_{1,4 ; \Omega}\left\|\mathbf{d}_{h}\right\|_{0,4 ; \Omega} \\
& \leq C_{9} h^{1 / 4}|\mathbf{w}|_{1, \Omega}\left|\mathbf{v}_{h}\right|_{1,4 ; \Omega}\left|\mathbf{d}_{h}\right|_{1 ; \Omega} \\
& \leq C_{10} h^{1 / 4}|\mathbf{w}|_{1, \Omega}\left|\mathbf{v}_{h}\right|_{1,4 ; \Omega}\|F\|_{V_{2}^{\prime}} .
\end{aligned}
$$

Thus,

$$
\lim _{h \rightarrow 0} \mathbf{S}_{2 h}\left(\mathbf{d}_{h}, \mathcal{R}_{2 h}\left(\mathbf{W}_{h} \cdot \nabla \mathbf{v}_{h}\right)\right)=0 .
$$

To complete the proof, we combine the weak lower semicontinuity of the norm on reflexive Banach spaces with estimate (77) to derive estimate (66).

Remark 8. This result shows the adaptivity of the general formulation provided by the abstract method (10), and of its analysis technique in Sections 3 and 4. The two main contributions in the actual application are

- To derive a discrete inf-sup condition in $L^{\alpha}$ norm $(1<\alpha<2)$, and

- To prove that the stabilizing terms vanish in the limit $h \rightarrow 0$.

We have chosen piecewise affine elements for simplicity, but the same analysis applies to the general finite element spaces introduced in Section 2.

\section{Conclusion}

We have developed in this paper a systematic way to extend the standard stability analysis of mixed methods to stabilized methods. The stability of pressure discretization follows from an underlying discrete inf-sup condition. The stability of velocity discretization follows from a uniform separation property between standard finite element spaces and bubble finite element spaces.

We have proved the adaptivity of this technique by analyzing two non-standard situations (spectral element method and primitive equations of the ocean) by means of the same essential analysis.

Roughly speaking, we have found a way to extend to stabilized methods any stability-related property that one could prove for stable mixed methods, as there is always an underlying discrete inf-sup condition to each actual stabilized method.

\section{APPENDIX}

Proof of Lemma 1. This proof is based upon the following:

Lemma 8. Denote by $B_{j}(K), W_{j}(K)$, respectively, the affine-transformed of spaces $B_{j}^{*}, W_{j}^{*}$ on element $K$.

Then, $B_{j}(K)$ is a space of virtual bubbles of $\left[H_{0}^{1}(K)\right]^{d}$ reproducing the operator $\tau_{K} I_{K}$ on $W_{j}(K)$, with respect to the inner product $\mathbf{S}_{K}$.

Proof. We shall respectively denote by $(\cdot, \cdot)_{*}$ and $((\cdot, \cdot))_{*}$ the standard inner products in $\left[L^{2}\left(K^{*}\right)\right]^{d}$ and $\left[H_{0}^{1}\left(K^{*}\right)\right]^{d}$. Consider an element $K \in \mathcal{T}_{h}$. Given a function $\mathbf{v}^{*}$ defined on the reference element $K^{*}$, let us denote by $\hat{\mathbf{v}}$ its affine-transformed on $K, \hat{\mathbf{v}}=\mathbf{v}^{*} \circ\left(F_{K}\right)^{-1}$, where $F_{K}$ is the affine mapping that transforms $K^{*}$ on to $K$, i.e., $F_{K}\left(x^{*}\right)=A_{K} x^{*}+b_{K}$.

If $\mathbf{w}^{*}, \mathbf{v}^{*} \in\left[H_{0}^{1}\left(K^{*}\right)\right]^{d}$, then $\hat{\mathbf{w}}, \hat{\mathbf{v}} \in\left[H_{0}^{1}(K)\right]^{d}$ and

$$
\mathbf{S}_{K}(\hat{\mathbf{w}}, \hat{\mathbf{v}})_{K}=\left|\operatorname{det} A_{K}\right| \beta_{K} h_{K}^{-2}\left(\left(\mathbf{w}^{*}, \mathbf{v}^{*}\right)\right)_{*} .
$$


Denote by $\mathcal{R}^{*}$ the static condensation operator acting on space $B_{j}^{*}$, associated to the inner product $((\cdot, \cdot))_{*}$. Given $\mathbf{v}^{*} \in W_{j}^{*}, \mathcal{R}^{*}\left(\mathbf{v}^{*}\right)$ satisfies

$$
\left(\left(\mathcal{R}^{*}\left(\mathbf{v}^{*}\right), \zeta^{*}\right)\right)_{*}=\left(\mathbf{v}^{*}, \zeta^{*}\right)_{*}, \quad \forall \zeta^{*} \in B_{j}^{*}
$$

From (79) we deduce that for any $\mathbf{w} \in W_{j}(K)$,

$$
h_{K}^{2} \beta_{K}^{-1} \mathbf{S}_{K}\left(\widehat{\mathcal{R}^{*}\left(\mathbf{w}^{*}\right)}, \zeta\right)=(\mathbf{w}, \zeta)_{K}, \quad \forall \zeta \in B_{j}(K)
$$

where $\mathbf{w}^{*}=\mathbf{w} \circ F_{K}$. Then, $\mathcal{R}_{K}(\mathbf{w})=h_{K}^{2} \beta_{K}^{-1} \widehat{\mathcal{R}^{*}\left(\mathbf{w}^{*}\right)}$.

Furthermore, by hypothesis,

$$
\left(\left(\mathcal{R}^{*}\left(\mathbf{w}_{1}^{*}\right), \mathcal{R}^{*}\left(\mathbf{w}_{2}^{*}\right)\right)\right)_{*}=\tau^{*}\left(\mathbf{w}_{1}^{*}, \mathbf{w}_{2}^{*}\right)_{*}, \quad \forall \mathbf{w}_{1}^{*}, \mathbf{w}_{2}^{*} \in W_{j}^{*}
$$

From (79), this implies

$$
h_{K}^{2} \beta_{K}^{-1} \mathbf{S}_{K}\left(\widehat{\mathcal{R}^{*}\left(\mathbf{w}_{1}^{*}\right)}, \widehat{\mathcal{R}^{*}\left(\mathbf{w}_{2}^{*}\right)}\right)=\tau^{*}\left(\mathbf{w}_{1}, \mathbf{w}_{2}\right)_{K}, \quad \forall \mathbf{w}_{1}, \mathbf{w}_{2} \in W_{j}(K) .
$$

Thus,

$$
\mathbf{S}_{K}\left(\mathcal{R}_{K}\left(\mathbf{w}_{1}\right), \mathcal{R}_{K}\left(\mathbf{w}_{2}\right)\right)=\tau_{K}\left(\mathbf{w}_{1}, \mathbf{w}_{2}\right)_{K}, \quad \forall \mathbf{w}_{1}, \mathbf{w}_{2} \in W_{j}(K)
$$

Consider now an element $\varphi \in\left[H^{-1}(\Omega)\right]^{d}$. Then, $\mathcal{R}_{h}(\varphi)_{\left.\right|_{K}}=\mathcal{R}_{K}\left(\varphi_{\left.\right|_{K}}\right)$. This occurs because $\operatorname{int}(K) \cap \operatorname{int}\left(K^{\prime}\right)=\varnothing$ if $K \neq K^{\prime}$.

Then, if $\mathbf{w}_{1}, \mathbf{w}_{2} \in W_{h}^{(j)}$,

$$
\mathbf{S}_{h}\left(\mathcal{R}_{h}^{(j)}\left(\mathbf{w}_{1}\right), \mathcal{R}_{h}^{(j)}\left(\mathbf{w}_{2}\right)\right)=\sum_{K \in \mathcal{T}_{h}} \mathbf{S}_{K}\left(\mathcal{R}_{h}^{(j)}\left(\mathbf{w}_{\left.1\right|_{K}}\right), \mathcal{R}_{h}^{(j)}\left(\mathbf{w}_{\left.2\right|_{K}}\right)\right)=\sum_{K \in \mathcal{T}_{h}} \tau_{K}\left(\mathbf{w}_{2}, \mathbf{w}_{1}\right)_{K} .
$$

This last equality holds because of Lemma 8. To finish the proof, we observe that the orthogonal projection of $\mathbf{f}_{\left.\right|_{K}}$ on $W(K)$ with respect to the $L^{2}$ inner product is just $\mathbf{f}_{\left.h\right|_{K}}$. Then,

$$
\sum_{K \in \mathcal{T}_{h}} \tau_{K}\left(\mathbf{f}, \mathbf{w}_{1}\right)_{K}=\sum_{K \in \mathcal{T}_{h}} \tau_{K}\left(\mathbf{f}_{h}, \mathbf{w}_{1}\right)_{K}=\mathbf{S}_{h}\left(\mathcal{R}_{h}^{(j)}\left(\mathbf{w}_{1}\right), \mathcal{R}_{h}^{(j)}\left(\mathbf{f}_{h}\right)\right) .
$$

Proof of Lemma 2. Consider a triangulation $\mathcal{T}_{h}$ of the family. Given an element $K \in \mathcal{T}_{h}$, denote by $h_{K}$ the diameter of element $K$. Let us consider the following bilinear form on $\left[H_{0}^{1}(\Omega)\right]^{d}$,

$$
((\mathbf{w}, \mathbf{v}))_{h}=\sum_{K \in \mathcal{T}_{h}} \int_{K}\left(C_{K} \nabla \mathbf{w}\right): \nabla \mathbf{v} \mathrm{d} x, \quad \forall \mathbf{w}, \mathbf{v} \in\left[H_{0}^{1}(\Omega)\right]^{d} .
$$

Here, matrix $C_{K}$ is defined as in $(34)$. The form $((\cdot, \cdot))_{h}$ is an inner product on $\left[H_{0}^{1}(\Omega)\right]^{d}$, as each matrix $C_{K}$ is symmetric and positive definite.

Some standard estimates yield

$$
\left|((\mathbf{w}, \mathbf{v}))_{h}\right| \leq M_{h}|\mathbf{w}|_{1}|\mathbf{v}|_{1},((\mathbf{w}, \mathbf{w}))_{h} \geq \Lambda_{h}|\mathbf{w}|_{1}^{2},
$$

where $M_{h}=\frac{1}{h_{K}} \max _{K, l} \sigma_{l}^{K}, \Lambda_{h}=\frac{1}{h_{K}} \min _{K, l} \sigma_{l}^{K}, \sigma_{1}^{K}, \ldots, \sigma_{d}^{K}$ denoting the singular values of $A_{K}$. 
As the family of triangulations is regular, there exist two constants $C_{1}>0, C_{2}>0$ such that (cf. Ciarlet [13]),

$$
C_{1} h_{K} \leq \sigma_{l}^{K} \leq C_{2} h_{K}, \quad \forall K \in \mathcal{T}_{h}, \forall h>0, \forall l=1, \ldots, d
$$

Consequently, the forms $((\cdot, \cdot))_{h}$ define inner products on $\left[H_{0}^{1}(\Omega)\right]^{d}$ which are uniformly (in $h$ ) equivalent to the standard one. If we denote by $\|\cdot\|_{h}$ the norm generated by the inner product $((\cdot, \cdot))_{h}$, we have

$$
\Lambda|\mathbf{w}|_{1} \leq\|\mathbf{w}\|_{h} \leq M|\mathbf{w}|_{1}, \quad \forall \mathbf{w} \in\left[H_{0}^{1}(\Omega)\right]^{d} ; \quad \text { where } \quad \Lambda=C_{1}^{2}>0, \quad M=C_{2}^{2}>0
$$

Let us now make the change of variable $x=A_{K} x^{*}+b_{K}$. Given $\mathbf{w}, \mathbf{v} \in\left[H_{0}^{1}(\Omega)\right]^{d}$, define $\mathbf{w}^{K}\left(x^{*}\right)=\mathbf{w}(x)$, $\mathbf{v}^{K}\left(x^{*}\right)=\mathbf{v}(x), \forall x^{*} \in K^{*}$. Then,

$$
\int_{K}\left(C_{K} \nabla \mathbf{w}\right): \nabla \mathbf{v} \mathrm{d} x=h_{K}^{-2}\left|\operatorname{det} A_{K}\right| \int_{K^{*}} \nabla \mathbf{w}^{K}: \nabla \mathbf{v}^{K} \mathrm{~d} x^{*}
$$

Thus, given $\mathbf{y}_{h} \in Y_{h}, \mathbf{z}_{h} \in Z_{h}$, we have

$$
\left(\left(\mathbf{y}_{h}, \mathbf{z}_{h}\right)\right)_{h}=\sum_{K \in \mathcal{T}_{h}} h_{K}^{-2}\left|\operatorname{det} A_{K}\right| \int_{K^{*}} \nabla \mathbf{y}_{h}^{K}: \nabla \mathbf{z}_{h}^{K} \mathrm{~d} x^{*} .
$$

Notice that we always have $\mathbf{y}_{h}^{K} \in Y^{*}, \mathbf{z}_{h}^{K} \in Z^{*}$.

Consider now the bilinear form on $\left[H^{1}\left(K^{*}\right)\right]^{d}$,

$$
((\tilde{\mathbf{w}}, \tilde{\mathbf{v}}))_{*}=\int_{K^{*}} \nabla \tilde{\mathbf{w}}: \nabla \tilde{\mathbf{v}} \mathrm{d} x^{*}
$$

This is an inner product on the quotient space $\tilde{H}=\left[H^{1}\left(K^{*}\right)\right]^{d} / \mathbf{R}$. The spaces $\tilde{Y}^{*}=Y^{*} / \mathbf{R}$ and $\tilde{Z}^{*}=Z^{*} / \mathbf{R}$ are subspaces of $\tilde{H}$ satisfying $\tilde{Y}^{*} \cap \tilde{Z}^{*}=\{0\}$.

Consider now a fixed nonzero $\tilde{\mathbf{z}} \in \tilde{Z}^{*}$. Denote by $\Pi$ the orthogonal projection from $\tilde{H}$ on to $\tilde{Y}^{*}$ with respect to the inner product $((\cdot, \cdot))_{*}$. Then,

$$
-\|\tilde{\mathbf{y}}\|_{*}\|\Pi \tilde{\mathbf{z}}\|_{*} \leq((\tilde{\mathbf{y}}, \tilde{\mathbf{z}}))_{*} \leq\|\tilde{\mathbf{y}}\|_{*}\|\Pi \tilde{\mathbf{z}}\|_{*}, \quad \forall \tilde{\mathbf{y}} \in \tilde{Y}^{*}
$$

where $\|\cdot\|_{*}$ denotes the norm associated to the inner product $((\cdot, \cdot))_{*}$. As $\tilde{Y}^{*} \cap \tilde{Z}^{*}=\{0\}$, $\tilde{\mathbf{z}}$ cannot belong to $\tilde{Y}^{*}$ and $\|\Pi \tilde{\mathbf{z}}\|_{*}<\|\tilde{\mathbf{z}}\|_{*}$. Thus, there exists a constant $\delta>0$ such that

$$
\left|((\tilde{\mathbf{y}}, \tilde{\mathbf{z}}))_{*}\right| \leq(1-\delta)\|\tilde{\mathbf{y}}\|_{*}\|\tilde{\mathbf{z}}\|_{*}, \quad \forall \tilde{\mathbf{y}} \in \tilde{Y}^{*}
$$

Define $N=\sup \left\{\left|((\tilde{\mathbf{y}}, \tilde{\mathbf{z}}))_{*}\right|\right.$ for $\tilde{\mathbf{y}} \in \tilde{Y}^{*}, \tilde{\mathbf{z}} \in \tilde{Z}^{*}$ with $\left.\|\tilde{\mathbf{y}}\|_{*}=\|\tilde{\mathbf{z}}\|_{*}=1\right\}$. As both $\tilde{Y}^{*}$ and $\tilde{Z}^{*}$ are spaces of finite dimension, in fact this supremum is achieved. Due to (83), we should have $N<1$. Thus, we may assume that (83) holds for all $\tilde{\mathbf{y}} \in \tilde{Y}^{*}$ and for all $\tilde{\mathbf{z}} \in \tilde{Z}^{*}$.

From (82) we now obtain

$$
\begin{aligned}
\left|\left(\left(\mathbf{y}_{h}, \mathbf{z}_{h}\right)\right)_{h}\right| & \leq(1-\delta) \sum_{K \in \mathcal{T}_{h}} h_{K}^{-2}\left|\operatorname{det} A_{K}\right|\left(\int_{K^{*}}\left|\nabla \mathbf{y}_{h}^{K}\right|^{2} \mathrm{~d} x^{*}\right)^{1 / 2}\left(\int_{K^{*}}\left|\nabla \mathbf{z}_{h}^{K}\right|^{2} \mathrm{~d} x^{*}\right)^{1 / 2} \\
& \leq(1-\delta)\left\|\mathbf{y}_{h}\right\|_{h}\left\|\mathbf{z}_{h}\right\|_{h} .
\end{aligned}
$$


Then,

$$
\begin{aligned}
\left\|\mathbf{y}_{h}+\mathbf{z}_{h}\right\|_{h}^{2} & \geq\left\|\mathbf{y}_{h}\right\|_{h}^{2}+\left\|\mathbf{z}_{h}\right\|_{h}^{2}-2(1-\delta)\left\|\mathbf{y}_{h}\right\|_{h}\left\|\mathbf{z}_{h}\right\|_{h} \\
& \geq \delta\left(\left\|\mathbf{y}_{h}\right\|_{h}^{2}+\left\|\mathbf{z}_{h}\right\|_{h}^{2}\right)
\end{aligned}
$$

From this last inequality,

$$
\left\|\mathbf{y}_{h}\right\|_{h}+\left\|\mathbf{z}_{h}\right\|_{h} \leq \sqrt{\frac{2}{\delta}}\left\|\mathbf{y}_{h}+\mathbf{z}_{h}\right\|_{h}
$$

Now, it is enough to use the uniform coerciveness and boundedness of the inner products $((\cdot, \cdot))_{h}$ to conclude the proof.

Proof of Lemma 4. The elements of $\left[Q_{N}\left(K^{*}\right)\right]^{d}$ are elements of $\left[H^{-1}\left(K^{*}\right)\right]^{d}$ if we identify the $H_{0}^{1}-H^{-1}$ duality with the $L^{2}$ inner product. Observe that the bilinear form $(\cdot, \cdot)_{N, K^{*}}$ is an inner product on $\left[Q_{N}\left(K^{*}\right)\right]^{d}$. Indeed, if $\left(\hat{q}_{N}, \hat{q}_{N}\right)_{N, K^{*}}=0$ for some $\hat{q}_{N} \in Q_{N}\left(K^{*}\right)$, then $\hat{q}_{N}\left(\xi_{i}, \xi_{j}, \xi_{k}\right)=0$, for $i, j, k=1, \cdots, N+1$. Then, $\hat{q}_{N}=0$.

Thus, similarly to the Step 1 in the proof of Theorem 2, by Theorem 4 , there exists a $\hat{\mu}>0$ such that if $0<\tau^{*} \leq \hat{\mu}$, there exists a finite-dimensional subspace $B^{*}$ of $\left[H_{0}^{1}\left(K^{*}\right)\right]^{d}$ that reproduces the operator $\tau^{*} I$ on $\left(\left[Q_{N}\left(K^{*}\right)\right]^{d},(\cdot, \cdot)_{N, K^{*}}\right)$ with respect to the standard inner product of $\left[H_{0}^{1}\left(K^{*}\right)\right]^{d}$.

Consider now an element $K \in \mathcal{T}_{h}$, and two functions $\mathbf{v}, \mathbf{w} \in\left[C^{0}(\Omega)\right]^{d}$. Define $\hat{\mathbf{v}}=\mathbf{v} \circ F_{K}, \hat{\mathbf{w}}=\mathbf{w} \circ F_{K}$, where $F_{K}$ is a bijective affine transformation from $K^{*}$ on to $K$. Then,

$$
(\mathbf{v}, \mathbf{w})_{N, K}=\left|\operatorname{det} A_{K}\right|(\hat{\mathbf{v}}, \hat{\mathbf{w}})_{N, K^{*}}, \quad A_{K}=\nabla F_{K}
$$

Denote by $B_{j}(K)$ the affine-transformed of $B^{*}$ by $F_{K}^{-1}: B_{j}(K)=B^{*} \circ F_{K}^{-1}$. Similarly to Lemma 8, we deduce that $B_{j}(K)$ is a space of virtual bubbles that reproduces the operator $\tau_{K} I$ on $\left(\left[Q_{N}(K)\right]^{d},(\cdot, \cdot)_{N, K}\right)$ with respect to the inner product $\mathbf{S}_{K}$ defined by (34) with $\beta_{K}=\tau^{*} \tau_{K}^{-1} h_{K}^{2}$.

Denote also by $B_{\mathcal{H}}$ the finite element subspace of $\left[H_{0}^{1}(\Omega)\right]^{d}$ generated by the reference element $B^{*}$ on triangulation $\mathcal{T}_{h}$. Similarly to Lemma 1 , we deduce that

$$
\mathbf{S}_{h}\left(\mathcal{R}_{\mathcal{H}}\left(\mathbf{w}_{1}\right), \mathcal{R}_{\mathcal{H}}\left(\mathbf{w}_{2}\right)\right)=\sum_{K \in \mathcal{T}_{h}} \tau_{K}\left(\mathbf{w}_{1}, \mathbf{w}_{2}\right)_{N, K}, \quad \forall \mathbf{w}_{1}, \mathbf{w}_{2} \in W_{\mathcal{H}}
$$

where $\mathcal{R}_{\mathcal{H}}$ denotes the static condensation operator on $B_{\mathcal{H}}$ with respect to form $\mathbf{S}_{h}$.

Consider now $\mathbf{v}_{1}, \mathbf{v}_{2} \in\left[C_{p, h}(\Omega)\right]^{d}$. Then, $I_{\mathcal{H}} \mathbf{v}_{i} \in W_{\mathcal{H}}, i=1,2$ and $\left(I_{\mathcal{H}} \mathbf{v}_{1}, I_{\mathcal{H}} \mathbf{v}_{2}\right)_{N, K}=\left(\mathbf{v}_{1}, \mathbf{v}_{2}\right)_{N, K}$. Consequently, (45) holds.

Proof of Lemma \% i) As $Z^{*}$ does not contain the constant functions, then the $H^{1}$ seminorm is a norm on $Z^{*}$. Therefore, there exists a constant $C_{*}>0$ such that

$$
\left\|\mathbf{z}^{*}\right\|_{0} \leq C_{*}\left|\mathbf{z}^{*}\right|_{1}, \quad \forall Z^{*} \in Z^{*}
$$

Consider now $\mathbf{z}_{h} \in Z_{h}$. Then, using the notation introduced in Lemma 2,

$$
\left\|\mathbf{z}_{h}\right\|_{0}^{2}=\sum_{K \in \mathcal{T}_{h}}\left|\operatorname{det} A_{K}\right|\left\|\mathbf{z}_{h}^{K}\right\|_{0, K^{*}}^{2} \leq C_{*} \sum_{K \in \mathcal{T}_{h}}\left|\operatorname{det} A_{K}\right|\left|\mathbf{z}_{h}^{K}\right|_{1, K^{*}}^{2}
$$

Following Girault and Raviart [17], Lemma A.1, there exists a constant $\gamma>0$ such that

$$
\left|\operatorname{det} A_{K}\right|^{1 / 2}\left|\mathbf{z}_{h}^{K}\right|_{1, K^{*}} \leq \gamma\left\|A_{K}\right\|\left|\mathbf{z}_{h}\right|_{1, K}
$$


where $\left\|A_{K}\right\|$ denotes the spectral matrix norm. Then,

$$
\left\|\mathbf{z}_{h}\right\|_{0}^{2} \leq C_{*} \gamma^{2} \sum_{K \in \mathcal{T}_{h}}\left\|A_{K}\right\|^{2}\left|\mathbf{z}_{h}\right|_{1, K}^{2} \leq C_{1} h^{2}\left|\mathbf{z}_{h}\right|_{1}^{2},
$$

for some constant $C_{1}>0$.

Let us now consider the interpolation estimate (cf. Brézis [6]),

$$
\|\mathbf{w}\|_{0, q} \leq C_{2}\|\mathbf{w}\|_{0}^{\beta}\|\mathbf{w}\|_{0,6}^{1-\beta}, \quad \forall \mathbf{w} \in\left[L^{6}(\Omega)\right]^{d},
$$

if $2 \leq q \leq 6$, with $\beta$ given in (78). As $\left[H_{0}^{1}(\Omega)\right]^{d}$ is continuously embedded in $\left[L^{6}(\Omega)\right]^{d}$ if $d=2$ or $d=3$, then (78) follows.

ii) Consider a sequence $\left\{\mathbf{z}_{h}\right\}_{h>0}$, with $\mathbf{z}_{h} \in Z_{h}$. This sequence contains a subsequence, that we still denote in the same way, weakly convergent to some element $\mathbf{z}$ in $\left[H_{0}^{1}(\Omega)\right]^{d}$. As $\left[H_{0}^{1}(\Omega)\right]^{d}$ is compactly embedded in $\left[L^{2}(\Omega)\right]^{d}$, we may assume that this sequence converges strongly in $\left[L^{2}(\Omega)\right]^{d}$.

Recall that space $Y_{h}^{(0)}$ is defined by

$$
Y_{h}^{(0)}=\left\{\mathbf{v} \in\left[L^{2}(\Omega)\right]^{d} \mid \mathbf{v}_{\left.\right|_{K}} \text { is constant, } \quad \forall K \in \mathcal{T}_{h}\right\} .
$$

Due to standard finite element interpolation analysis, there exists a sequence $\left\{\mathbf{y}_{h}\right\}_{h>0}$, with $\mathbf{y}_{h} \in Y_{h}^{(0)}$, strongly convergent to $\mathbf{z}$ in $\left[L^{2}(\Omega)\right]^{d}$ (even if the family of triangulations is not regular).

Denote by $Y^{*}$ the reference space that generates space $Y_{h}^{(0)}$. By hypothesis, $Y^{*} \cap Z^{*}=\{0\}$. Then, there exists $\rho>0$ such that

$$
\left|\left(\mathbf{z}^{*}, \mathbf{y}^{*}\right)_{K^{*}}\right| \leq(1-\rho)\left\|\mathbf{z}^{*}\right\|_{0, K^{*}}\left\|\mathbf{y}^{*}\right\|_{0, K^{*}}, \quad \forall \mathbf{z}^{*} \in Z^{*}, \mathbf{y}^{*} \in Y^{*} .
$$

This is proved similarly to estimate (83) in Lemma 2. Thus,

$$
\begin{aligned}
\left|\left(\mathbf{z}_{h}, \mathbf{y}_{h}\right)\right| & =\sum_{K \in \mathcal{T}_{h}}\left|\operatorname{det} A_{K}\right|\left|\left(\mathbf{z}_{h}^{K}, \mathbf{y}_{h}^{K}\right)_{K^{*}}\right| \leq(1-\rho) \sum_{K \in \mathcal{T}_{h}}\left|\operatorname{det} A_{K}\right|\left\|\mathbf{z}_{h}^{K}\right\|_{0, K^{*}}\left\|\mathbf{y}_{h}^{K}\right\|_{0, K^{*}} \\
& \leq(1-\rho)\left\|\mathbf{z}_{h}\right\|_{0}\left\|\mathbf{y}_{h}\right\|_{0} .
\end{aligned}
$$

Consequently, $\mathbf{z}=0$ as $\|\mathbf{z}\|_{0}^{2}=\lim _{h \rightarrow 0}\left|\left(\mathbf{z}_{h}, \mathbf{y}_{h}\right)\right| \leq(1-\rho)\|\mathbf{z}\|_{0}^{2}$.

As the limit of any weakly convergent subsequence is necessarily zero, the the whole sequence $\left\{\mathbf{z}_{h}\right\}_{h>0}$ converges weakly to zero.

\section{REFERENCES}

[1] C. Amrouche and V. Girault, Decomposition of Vector spaces and application to the Stokes problem in arbitrary dimensions. Czeschoslovak Math. J. 44 (1994) 109-140.

[2] O. Besson and M. R. Laydi, Some estimates for the anisotropic Navier- Stokes equations and for the hydrostatic approximation. RAIRO-Modél. Math. Anal. Numér. 26 (1992) 855-865.

[3] I. Babuška, The Finite Element Method with Lagrange multipliers. Numer. Math. 20 (1973) 179-192.

[4] C. Baiocchi, F. Brezzi and L. P. Franca, Virtual Bubbles and Galerkin-least-squares type methods (Ga.L.S.). Comput. Methods Appl. Mech. Engrg. 105 (1993) 125-141.

[5] C. Bernardi and Y. Maday, Approximations spectrales de problèmes aux limites elliptiques. Springer-Verlag, Berlin (1992).

[6] H. Brézis, Analyse Fonctionnelle. Masson, Paris (1983).

[7] F. Brezzi, On the existence, uniqueness and approximation of saddle-point problems arising from Lagrange Multipliers. RAIROAnal. Numér. R2 (1974) 129-151.

[8] F. Brezzi and J. Douglas, Stabilized mixed methods for the Stokes problem. Numer. Math. 53 (1988) 225-236. 
[9] F. Brezzi and J. Pitkäranta, On the stabilization of Finite Element approximations of the Stokes problem, in Efficient Solutions for Elliptic Systems. Notes on Numerical Fluid Mechanics 10, W. Hackbusch Ed., Springer-Verlag, Berlin (1984) 11-19.

[10] T. Chacón Rebollo, A term by term Stabilization Algorithm for Finite Element solution of incompressible flow problems. Numer. Math. 79 (1998) 283-319.

[11] T. Chacón Rebollo and A. Domínguez Delgado, A unified analysis of Mixed and Stabilized Finite Element Solutions of Navier-Stokes equations. Comput. Methods Appl. Mech. Engrg. 182 (2000) 301-331.

[12] T. Chacón Rebollo and F. Guillén González, An intrinsic analysis of existence of solutions for the hydrostatic approximation of Navier-Stokes equations. C. R. Acad. Sci. Paris, Série I 330 (2000) 841-846.

[13] P.G. Ciarlet, The Finite Element Method for Elliptic Problems. North-Holland, Amsterdam (1978).

[14] L.P. Franca and S.L. Frey, Stabilized Finite Elements: II. The incompressible Navier-Stokes equations. Comput. Methods Appl. Mech. Engrg. 99 (1992) 209-233.

[15] L.P. Franca and R. Stenberg, Error analysis fo some Galerkin-Least-Squares methods for the elasticity equations. SIAM J. Numer. Anal. 28 (1991) 1680-1697.

[16] L.P. Franca, T.J.R. Hughes and R. Stenberg, Stabilized Finite Element Methods, in Incompressible Computational Fluid Dynamics, M.D. Gunzburger and R.A. Nicolaides Eds., Cambridge Univ. Press, New York (1993).

[17] V. Girault and P.A. Raviart, Finite Element Methods for Navier-Stokes equations. Springer-Verlag, Berlin (1988).

[18] R. Dautray and L.L. Lions, Analyse Mathématique et Calcul Numérique pour les Sciences et les Techniques. Masson, Paris (2000).

[19] P. Gervasio and F. Saleri, Stabilized Spectral Element approximation for the Navier-Stokes equations. Numer. Methods Partial Differential Eq. 14 (1988) 115-141.

[20] T.J.R. Hughes and L.P. Franca, A new Finite Element formulation for CFD: VII. The Stokes problem with various well-posed boundary conditions: Symmetric formulations that converge for all velocity/pressure spaces. Comput. Methods Appl. Mech. Engrg. 65 (1987) 85-96.

[21] T.J.R. Hughes, L.P. Franca and M. Balestra, A new Finite Element formulation for CFD: V. Circumventing the Brezzi-Babuška condition: A stable Petrov-Galerkin formulation of the Stokes problem accommodating equal-order interpolations. Comput. Methods Appl Mech. Engrg. 59 (1986) 85-99.

[22] P. Knobloch and L. Tobiska, Stabilization methods of Bubble type for the $Q_{1}-Q_{1}$-Element applied to the incompressible Navier-Stokes equations. ESAIM: M2AN 34 (2000) 85-107.

[23] R. Lewandowski, Analyse Mathématique et Océanographie. Masson, Paris (1997).

[24] J.L. Lions, R. Temam and S. Wang, New formulation of the primitive equations of the atmosphere and applications. Nonlinearity 5 (1992) 237-288.

[25] R. Pierre, Simple $C^{0}$-approximations for the computation of incompressible flows. Comput. Methods Appl Mech. Engrg. 68 (1989) 205-228.

[26] G. Russo, Bubble stabilization of Finite Element Methods fo the linearized incompressible Navier-Stokes equations. Comput. Methods Appl Mech. Engrg. 132 (1996) 335-343.

[27] L. Tobishka and R. Verfürth, Analysis of a Streamline Diffusion finite element method for the Stokes and Navier-Stokes equations. SIAM J. Numer. Anal. 33 (1996) 107-127.

[28] R. Verfürth, Analysis of some Finite Element solutions for the Stokes Problem. RAIRO-Anal. Numér. 18 (1984) 175-182.

To access this journal online:

www.edpsciences.org 